GB

$$
\begin{aligned}
& \text { GANIL-P } 94.68 \\
& \text { Sce } 94.16
\end{aligned}
$$
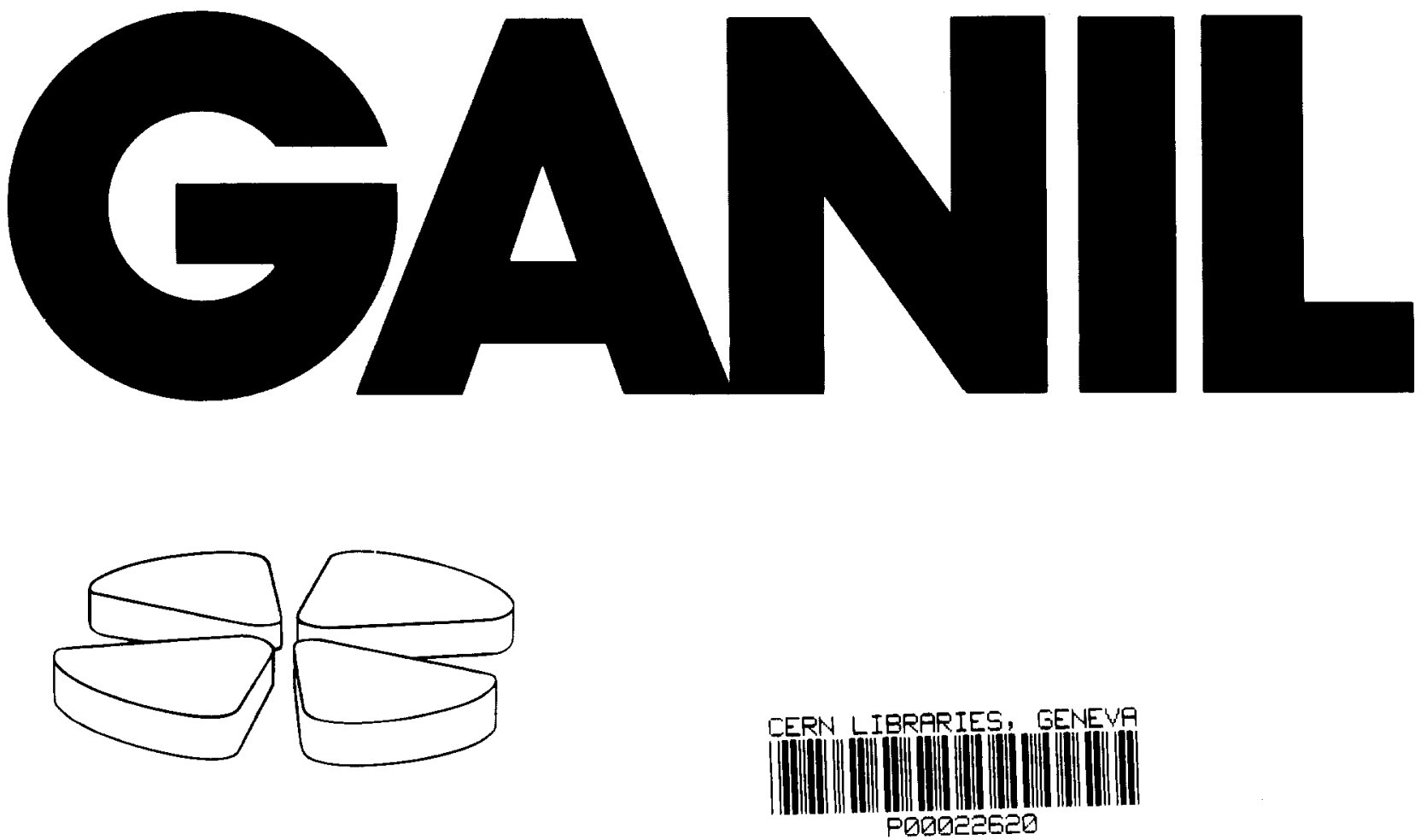

Extensions of the Interacting Boson Model

$$
\text { P Van Isacker }{ }^{1,2,3)} \text { and D D Warner }{ }^{1)}
$$

1) SERC Daresbury Laboratory, Warrington WA4 4AD, England

2) Department of Physics, University of Surrey, Guildford GU2 5XH, England 3) GANIL, BP 5027, F-14021 Caen Cedex, France

To appear as a Topical Review in Journal of Physics $G$ 



\title{
Extensions of the Interacting Boson Model
}

\author{
P Van Isacker ${ }^{1,2,3)}$ and D D Warner ${ }^{1)}$ \\ 1) SERC Daresbury Laboratory, Warrington WA4 4AD, England \\ 2) Department of Physics, University of Surrey, Guildford GU2 5XH, England \\ 3) GANIL, BP 5027, F-14021 Caen Cedex, France
}

\begin{abstract}
A review is given of some of the more topical extensions of the Interacting Boson Model of collective nuclear excitations. After introducing some elementary and basic ideas which illuminate the general principles of the algebraic approach, their application to nuclear structure in the context of the various extensions of the Interacting Boson Model is presented: the introduction of the neutron-proton degree of freedom and its relevance to a recently discovered class of collective states (so-called 'scissors' states); the coupling of a fermion to a system of interacting bosons to describe nuclei with odd numbers of neutrons and/or protons; and the inclusion of the isospin degree of freedom in the model, necessary in light nuclei where neutrons and protons occupy the same valence shell. These different developments are summarised with special emphasis on the new features and quantum numbers appearing specifically as a result of the algebraic method. The final part of this paper reviews a general algebraic framework for describing scattering phenomena, which is inspired by the methods used in structure phenomena.
\end{abstract}

\section{Introduction}

The Interacting Boson Model (IBM) has been broadened and developed steadily since its inception (Arima and Iachello, 1975) some two decades ago so that, by now, it consists of a family of nuclear structure models linked by common underlying assumptions concerning their microscopic foundation and by the algebraic origin of their formalism. In a more general context, the IBM represents one of the three principal approaches to the description of nuclear structure. The first centres on the shell model, either in its 
pure form, or in the guise of one of the many offshoots and approximations which have been developed to deal with nuclei that are not near enough to closed shells to render an exact diagonalisation tractable. The second is the geometrical model, in which an average shape of the nucleus is proposed and the spectrum of possible excitations of that shape are considered. Of course, the concept of the mean field underpins and links these two frameworks. The third method is the algebraic approach, where the focus is on the search for symmetries in the quantal states of the nuclear system. The insights which result from recognising the existence and role of symmetries underlying the nuclear structure problem can be profound and have frequently resulted in a considerable enhancement of our understanding as well as in the prediction of new phenomena. However, links to the other two frameworks often have to be forged to provide this understanding since the algebraic approach sometimes results in a solution without an immediately obvious physical interpretation. This is hardly surprising since the symmetries relevant to the nuclear structure problem describe particular states of motion of the system and their appearance depends on the algebraic properties of a set of operators, rather than on the actual nature of the constituents of the system. Thus the methods and symmetries described here to treat collective features in the realm of nuclear structure are also relevant in the molecular regime and in particle physics. Although the constituents change, the operators describing them have the same algebraic characteristics and hence the symmetry properties persist.

The aim of this review is to show how the algebraic approach has been applied in recent years to many different aspects of the description of collective states in nuclei through the various extensions of the IBM. A particular effort is made to show how the same algebraic properties can appear in very different contexts and special emphasis is placed on the new features and quantum numbers which appear specifically as a result of the algebraic basis of the model and its associated recognition of symmetries. Although the IBM is undoubtedly the most extensively studied algebraic model to date, it should also be made clear that there are other algebraic approaches to collective structure which are being actively studied, such as the pseudo-SU(3) model (Ratna Raju et al., 1973) or the Fermion Dynamical Symmetry Model (Wu et al., 1986). While the details are very different, the basic principles of the methods are very similar.

In the next section we begin by giving an introduction to the algebraic method, illustrated by some familiar examples in nuclear and particle physics. We then deal with the application of the IBM in domains of ever-increasing complexity, beginning briefly with the simplest framework which treats the nucleus in terms of a single type of nucleon, 
and, moving on through the neutron-proton problem, the structure of odd-mass nuclei and the explicit inclusion of isospin. We finish with a discussion of one of the most recent major extensions of the technique to the description of scattering problems.

\section{The algebraic approach}

In this section we introduce some of the ideas and concepts which we believe to be of central importance to the algebraic approach (Frank and Van Isacker, 1994). They are long since known to physicists and, although the examples given in this section are mainly taken from nuclear and particle physics, have found repeated and fruitful applications in virtually all branches of physics.

We begin with some elementary considerations related to symmetry, mainly to introduce the notation we adopt in this section. For applications in low-energy nuclear physics, the idea of symmetry is most conveniently introduced via a Hamiltonian formalism. A Hamiltonian $H$, invariant under a set of transformations $g_{i}$ which together form a Lie group $G$, i.e.

$$
\left[H, g_{i}\right]=0 \quad \text { for } g_{i} \in G
$$

is said to have a symmetry $G$ or, alternatively, to be invariant under $G$. A well-known consequence of a symmetry is the occurrence of degeneracies in the eigenspectrum of $H$. Given an eigenstate $\psi$ of $H$ with energy $E$, the condition (2.1) immediately implies that the states $g_{i} \psi$ have the same energy. This enables one to write an arbitrary eigenstate of $H$ as $|\Gamma \gamma\rangle$, where the first quantum number $\Gamma$ is different for states with different energies and the second quantum number $\gamma$ is needed to distinguish degenerate eigenstates. The energy eigenvalues of a Hamiltonian satisfying (2.1) thus depend only on $\Gamma$,

$$
H|\Gamma \gamma\rangle=E(\Gamma)|\Gamma \gamma\rangle
$$

and, furthermore, the transformations $g_{i}$ do not admix states with different $\Gamma$ :

$$
g_{i}|\Gamma \gamma\rangle=\sum_{\gamma^{\prime}} a_{\gamma, \gamma^{\prime}}^{i, \Gamma}\left|\Gamma \gamma^{\prime}\right\rangle
$$

In the language of group theory the transformation coefficients $a_{\gamma, \gamma^{\prime}}^{i, \Gamma}$, when considered as matrices in the indices $\gamma$ and $\gamma^{\prime}$, provide a (matrix) representation of the group elements $g_{i}$ in the vector space spanned by the states $|\Gamma \gamma\rangle$. A representation obviously depends on $\Gamma$ and is denoted as $[\Gamma]$. Another ingredient borrowed from group theory concerns the construction of operators like $H$ in (2.1) that commute with all elements of $G$. Such 
operators are called Casimir operators and are denoted here as $C_{n}[G]$, the index $n$ referring to the order of the operator in the $g_{i}$. The enumeration of independent Casimir operators associated with a given group $G$ and the derivation of expressions for their eigenvalues $E_{n}(\Gamma)$, i.e.

$$
C_{n}[G]|\Gamma \gamma\rangle=E_{n}(\Gamma)|\Gamma \gamma\rangle
$$

is a well-studied problem, the solution of which can be found in many monographs on group theory (see, e.g. Wybourne, 1974).

Next we introduce the concept of dynamical symmetry, ${ }^{1}$ for which we need (at least) two groups $G_{1}$ and $G_{2}$ with $G_{1} \supset G_{2}$. We impose the condition of $G_{1}$ symmetry on the Hamiltonian $H$ and, as before, its eigenstates can be labelled as $\left|\Gamma_{1} \gamma_{1}\right\rangle$. But, since $G_{1} \supset G_{2}$, a Hamiltonian with $G_{1}$ symmetry necessarily must also have a symmetry $G_{2}$ and, consequently, its eigenstates can also be labelled as $\left|\Gamma_{2} \gamma_{2}\right\rangle$. Combination of the two properties leads to the eigenequation

$$
H\left|\Gamma_{1} \Gamma_{2} \gamma_{2}\right\rangle=E\left(\Gamma_{1}\right)\left|\Gamma_{1} \Gamma_{2} \gamma_{2}\right\rangle
$$

where the role of $\gamma_{1}$ is played by $\Gamma_{2} \gamma_{2}$ and hence the eigenvalues depend only on $\Gamma_{1}{ }^{2}$ The meaning of the labels used in (2.5) is further illustrated with the transformation properties of the states $\left|\Gamma_{1} \Gamma_{2} \gamma_{2}\right\rangle$ under the action of an element belonging to $G_{1}$ or $G_{2}$ :

$$
\begin{aligned}
& g_{i}\left|\Gamma_{1} \Gamma_{2} \gamma_{2}\right\rangle=\sum_{\Gamma_{2}^{\prime} \gamma_{2}^{\prime}} a_{\Gamma_{2} \gamma_{2}, \Gamma_{2}^{\prime} \gamma_{2}^{\prime}}^{i, \Gamma_{1}}\left|\Gamma_{1} \Gamma_{2}^{\prime} \gamma_{2}^{\prime}\right\rangle \quad \text { for } g_{i} \in G_{1} \\
& g_{i}\left|\Gamma_{1} \Gamma_{2} \gamma_{2}\right\rangle=\sum_{\gamma_{2}^{\prime}} a_{\gamma_{2}, \gamma_{2}^{\prime}}^{i, \Gamma_{2}}\left|\Gamma_{1} \Gamma_{2} \gamma_{2}^{\prime}\right\rangle \quad \text { for } g_{i} \in G_{2} .
\end{aligned}
$$

In many applications the condition of $G_{1}$ symmetry is found to be too strong and must be relaxed. A possible breaking of the $G_{1}$ symmetry occurs via the Hamiltonian

$$
H^{\prime}=a C_{n_{1}}\left[G_{1}\right]+b C_{n_{2}}\left[G_{2}\right]
$$

The essential idea is to take a combination of Casimir operators of $G_{1}$ and $G_{2}$. In (2.7) this combination is assumed to be a linear one but in general any (analytic) function of Casimir operators of $G_{1}$ and $G_{2}$ can be taken. Let us now look at the symmetry properties

\footnotetext{
${ }^{1}$ We follow the nomenclature and conventions adopted in the IBM.

${ }^{2}$ In (2.5) we have excluded the possibility that the same representation $\left[\Gamma_{2}\right]$ occurs more than once in $\left[\Gamma_{1}\right]$, in which case we would need an additional quantum number $\alpha$ to uniquely label the states as $\left|\Gamma_{1} \alpha \Gamma_{2} \gamma_{2}\right\rangle$. For the purpose of illustrating the concept of dynamical symmetry, however, we may ignore this technical complication.
} 
of the Hamiltonian $H^{\prime}$. Since $\left[H^{\prime}, g_{i}\right]=0$ for $g_{i} \in G_{2}, H^{\prime}$ is invariant under $G_{2}$. However, the Hamiltonian $H^{\prime}$ in general does not commute with all elements of $G_{1}$ and for this reason the $G_{1}$ symmetry is broken, the extent of the symmetry breaking depending on the ratio $b / a$. Furthermore, since $H^{\prime}$ is written as a combination of Casimir operators of $G_{1}$ and $G_{2}$, its eigenvalues are obtained in closed form:

$$
\left(a C_{n_{1}}\left[G_{1}\right]+b C_{n_{2}}\left[G_{2}\right]\right)\left|\Gamma_{1} \Gamma_{2} \gamma_{2}\right\rangle=\left(a E_{n_{1}}\left(\Gamma_{1}\right)+b E_{n_{2}}\left(\Gamma_{2}\right)\right)\left|\Gamma_{1} \Gamma_{2} \gamma_{2}\right\rangle
$$

Thus we conclude that, although $H^{\prime}$ is not invariant under $G_{1}$, its eigenstates are the same as those of $H$ in (2.5). The Hamiltonian $H^{\prime}$ is said to have $G_{1}$ as a dynamical symmetry. The essential feature is that, although the eigenvalues of $H^{\prime}$ depend on $\Gamma_{1}$ and $\Gamma_{2}$ (and hence $G_{1}$ is not a symmetry), the eigenstates do not change during the breaking of the $G_{1}$ symmetry: the dynamical symmetry breaking splits but does not admix the eigenstates.

These ideas can be illustrated with some well-known examples. The first is taken from nuclear physics and concerns isospin multiplets of nuclei. To describe a system of interacting neutrons and protons we might, in first approximation, assume the Hamiltonian to be isospin invariant, since that is a symmetry property which we believe to be valid for the strong interaction. In the notation introduced above, $G_{1}$ in this example is the isospin group $\mathrm{SU}_{\mathrm{T}}(2)$, consisting of the operators $T_{+}, T_{z}$ and $T_{-}$, and $G_{2}$ should be identified with $\mathrm{O}_{\mathrm{T}}(2)=\left\{T_{z}\right\}$, the projection operator on the $z$ axis in isospace. An isospin-invariant Hamiltonian commutes with $T_{+}, T_{z}$ and $T_{-}$, and hence the eigenstates $\left|T M_{\mathrm{T}}\right\rangle$ with fixed $T$ and $M_{\mathrm{T}}=-T,-T+1, \ldots,+T$ are degenerate in energy. (Compare with (2.2) by making the substitutions $\Gamma \rightarrow T$ and $\gamma \rightarrow M_{\mathrm{T}}$.) Unlike the strong interaction, the electromagnetic interaction is not isospin invariant and lifts the degeneracy of the states $\left|T M_{\mathrm{T}}\right\rangle$. We assume that this symmetry breaking occurs dynamically and, furthermore, it can be shown that, since the Coulomb force has a two-body character, the breaking term is at most quadratic in $T_{z}$ (Elliott and Dawber, 1979). Under these two restrictions, the energies of corresponding nuclear states belonging to the same isospin multiplet are given by

$$
E\left(M_{\mathrm{T}}\right)=a+b M_{\mathrm{T}}+c M_{\mathrm{T}}^{2}
$$

Two conclusions are obtained from these considerations. First, because the electromagnetic symmetry breaking is assumed to occur in a dynamical manner, eigenstates of the nuclear Hamiltonian have good $T$ and $M_{\mathrm{T}}$. Extensive tests, involving selection rules in E1 transitions, $\beta$-decay and nuclear-reaction processes, have shown this to be a good approximation, at least at low nuclear excitation energies (Bohr and Mottelson, 1969). Second, 


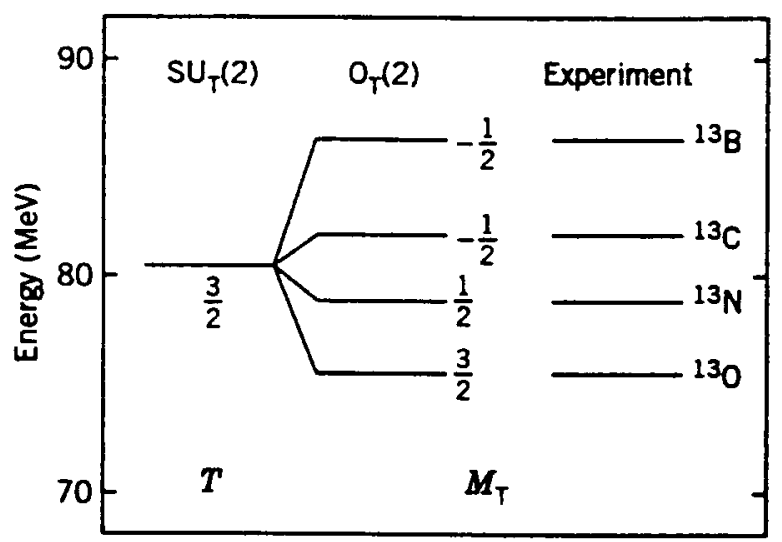

Figure 2.1: Binding energies of the $T=3 / 2$ isobaric analog states with $J^{\pi}=1 / 2^{-}$in ${ }^{13} \mathrm{~B},{ }^{13} \mathrm{C}$, ${ }^{13} \mathrm{~N}$ and ${ }^{13} \mathrm{O}$. The column on the left is obtained for an exact $\mathrm{SU}_{\mathrm{T}}(2)$ symmetry, which predicts states with different $M_{\mathrm{T}}$ to be degenerate. The middle column is obtained in the case of an $\mathrm{SU}_{\mathrm{T}}(2)$ dynamical symmetry, equation (2.9) with $a=80.59, b=-2.96$ and $c=-0.26$, in $\mathrm{MeV}$.

the two-body character of the Coulomb force leads to the expansion (2.9) for the energies of corresponding nuclear states with the same $T$. This formula can be tested for a $T=3 / 2$ multiplet consisting of isobaric analog states in ${ }^{13} \mathrm{~B},{ }^{13} \mathrm{C},{ }^{13} \mathrm{~N}$ and ${ }^{13} \mathrm{O}$. In figure 2.1 we plot the binding energies of the nuclei ${ }^{13} \mathrm{~B}$ and ${ }^{13} \mathrm{O}$, both of which have $T=\left|M_{\mathrm{T}}\right|=3 / 2$ in their ground state. The isobaric analog states in ${ }^{13} \mathrm{C}$ and ${ }^{13} \mathrm{~N}$ are $J^{\pi}=3 / 2^{-}$states at excitation energies of 15.11 and $15.07 \mathrm{MeV}$, respectively; these energies are substracted from the ground-state binding energies of ${ }^{13} \mathrm{C}$ and ${ }^{13} \mathrm{~N}$ to give the energies plotted in figure 2.1. In this example the energy splitting due to the Coulomb force clearly is well accounted for by the energy formula (2.9), which is perhaps not surprising since four data points are fitted with three parameters. However, we want to stress at this point that the quality of fits such as in figure 2.1 is not the most important aspect of dynamical symmetries, but rather the existence of good quantum numbers ( $T$ and $M_{\mathrm{T}}$ in this case).

The next example is taken from particle physics and concerns the classification of 'elementary' particles into $\mathrm{SU}(3)$ multiplets. In this case the relevant symmetry groups and their associated quantum numbers are

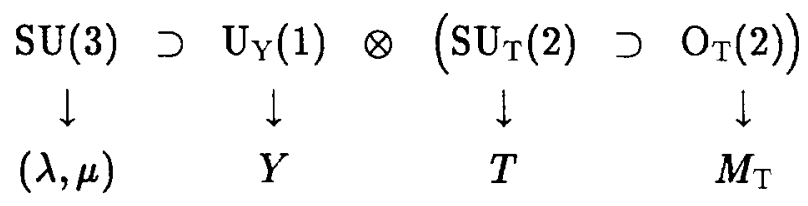

where $T$ and $M_{\mathrm{T}}$ are the isospin and its projection on the $z$ axis and $Y$ is the hypercharge. Instead of the notation $(\lambda, \mu)$, which we follow here, $\mathrm{SU}(3)$ representations often 
are denoted by their dimension, that is, the number of independent basis vectors in the representation (= the number of particles in the corresponding $\mathrm{SU}(3)$ multiplet). If we assume $\mathrm{SU}(3)$ invariance, all particles belonging to one multiplet are predicted to have the same mass. Since the observed masses differ by hundreds of $\mathrm{MeV}$, they clearly must contain SU(3)-symmetry breaking terms. However, $\mathrm{SU}(3)$ can be broken while maintaining good quantum numbers $Y, T$ and $M_{\mathrm{T}}$, that is, it can be broken dynamically. If we allow only up to quadratic terms, we find a mass operator of the form

$$
M=a+b C_{1}\left[\mathrm{U}_{\mathrm{Y}}(1)\right]+c C_{2}\left[\mathrm{U}_{\mathrm{Y}}(1)\right]+d C_{2}\left[\mathrm{SU}_{\mathrm{T}}(2)\right]+e C_{1}\left[\mathrm{O}_{\mathrm{T}}(2)\right]+f C_{2}\left[\mathrm{O}_{\mathrm{T}}(2)\right]
$$

with the eigenvalues

$$
M\left(Y, T, M_{\mathrm{T}}\right)=a+b Y+c Y^{2}+d T(T+1)+e M_{\mathrm{T}}+f M_{\mathrm{T}}^{2}
$$

Due to the electromagnetic interaction, $M$ is not scalar in isospin, but contains also isospin vector and tensor terms (two last terms in (2.11)), for the reasons we have referred to in the previous example. Similarly, one assumes the strong interaction to have a certain tensor character under $\mathrm{SU}(3)$ and this leads to a relation between the coefficients $c$ and $d$ in (2.12), resulting in the SU(3) mass-splitting formula (Okubo, 1962, Gell-Mann, 1962):

$$
M^{\prime}\left(Y, T, M_{\mathrm{T}}\right)=a+b Y+d\left(T(T+1)-\frac{1}{4} Y^{2}\right)+e M_{\mathrm{T}}+f M_{\mathrm{T}}^{2} .
$$

The process of successive symmetry breakings is illustrated in figure 2.2 with the example of the $\mathrm{SU}(3)$ octuplet $(\lambda, \mu)=(1,1)$, containing the neutron, the proton and the $\Lambda, \Sigma$ and $\Xi$ baryons.

We are now in a position to discuss one more concept frequently used in algebraic models, namely the one of a dynamical group, a single representation of which contains all states of the physical system under consideration. The idea is perhaps best understood with the help of the examples given above. For isobaric analog states in nuclei the dynamical group is $\mathrm{SU}_{\mathrm{T}}(2)$, since in that case we establish a relation between nuclear states contained in a single representation of $\mathrm{SU}_{\mathrm{T}}(2)$. The dynamical group in the particle physics example is $\mathrm{SU}(3)$, since all the particles which are simultaneously described belong to one $(\lambda, \mu)$ representation $((1,1)$ for the octuplet, $(3,0)$ for the decuplet, etc.). A persistent theme in physics has been the search for larger dynamical groups resulting in a more unified description of physical phenomena. This trend can be illustrated with figure 2.2. The near-equality of the masses of the neutron and the proton suggested the existence of isospin multiplets, later confirmed at higher energies for other 'elementary' 


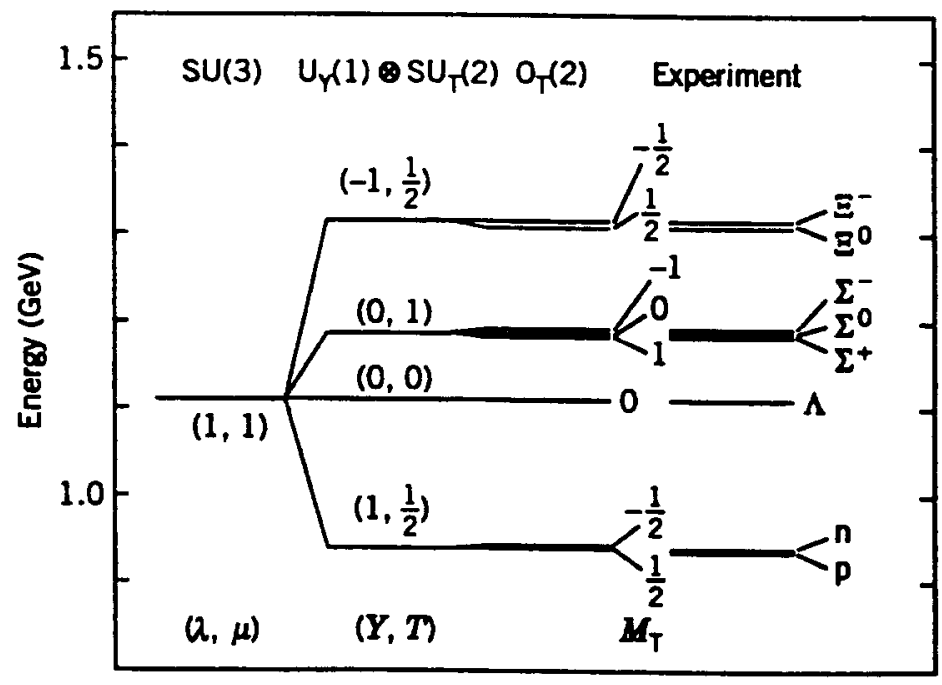

Figure 2.2: Mass spectrum of the $\mathrm{SU}(3)$ octuplet $(\lambda, \mu)=(1,1)$. The column on the left is obtained for an exact SU(3) symmetry, which predicts all masses to be the same, while the next two columns represent successive breakings of this symmetry in a dynamical manner. The column under $\mathrm{O}_{\mathrm{T}}(2)$ is obtained with (2.13) with $a=1111.3, b=-189.6, d=-39.9, e=-3.8$ and $f=0.9$, in $\mathrm{MeV}$.

particles. To establish, in turn, a relation between several of those multiplets, a larger dynamical group was needed, $\mathrm{SU}(3)$, which contains the isospin group $\mathrm{SU}_{\mathrm{T}}(2)$ as a subgroup. This unification process did not stop with $\mathrm{SU}(3)$ : to connect the different observed $(\lambda, \mu)$ multiplets, still larger dynamical groups have been proposed (SU(4),...). However, typically the symmetry associated with a larger dynamical group will be more strongly broken.

\section{Like-nucleon systems: IBM-1}

In the original version of the IBM, applicable to even-even nuclei, the basic building blocks are $s$ and $d$ bosons interacting via two-body forces, hence the name Interacting Boson Model. Unitary transformations among the six components occurring in the model ( $s^{\dagger}$ and $d_{\mu}^{\dagger}, \mu=0, \pm 1, \pm 2$ ) generate the group $\mathrm{U}(6)$, which thus plays the role of dynamical group, since all low-lying collective states of an even-even nucleus are described within a single (symmetric) representation $[N]$ of $\mathrm{U}(6)$, where $N$ is the number of bosons. Imposing a $\mathrm{U}(6)$ symmetry for the description of such states would constitute a poor approximation, since they would be predicted to be degenerate in energy. However, analogous to the examples given in section 2 , the $\mathrm{U}(6)$ symmetry can be broken in a dynamical manner. 
It has been shown by Arima and Iachello $(1976,1978,1979)$ that three different types of dynamical symmetries occur in the IBM, associated with the three reduction schemes

$$
\mathrm{U}(6) \supset\left\{\begin{array}{c}
\mathrm{U}(5) \supset \mathrm{O}(5) \\
\mathrm{SU}(3) \\
\mathrm{O}(6) \supset \mathrm{O}(5)
\end{array}\right\} \supset \mathrm{O}(3) \supset \mathrm{O}(2)
$$

where $\mathrm{O}(3)$ is the angular momentum group, consisting of the operators $L_{+}, L_{z}$ and $L_{-}$, and $\mathrm{O}(2)=\left\{L_{z}\right\}$. The Hamiltonians corresponding to these group chains are

$$
\begin{aligned}
H^{(\mathrm{I})} & =\epsilon C_{1}[\mathrm{U}(5)]+\alpha C_{2}[\mathrm{U}(5)]+\beta C_{2}[\mathrm{O}(5)]+\gamma C_{2}[\mathrm{O}(3)] \\
H^{(\mathrm{II})} & =\delta C_{2}[\mathrm{SU}(3)]+\gamma C_{2}[\mathrm{O}(3)] \\
H^{(\mathrm{III})} & =\eta C_{2}[\mathrm{O}(6)]+\beta C_{2}[\mathrm{O}(5)]+\gamma C_{2}[\mathrm{O}(3)]
\end{aligned}
$$

where the coefficients in front of the Casimir operators are named to conform with the notation introduced by Iachello and Arima (1987). For further reference throughout this paper we also give the quantum numbers appropriate for each of the classification schemes:

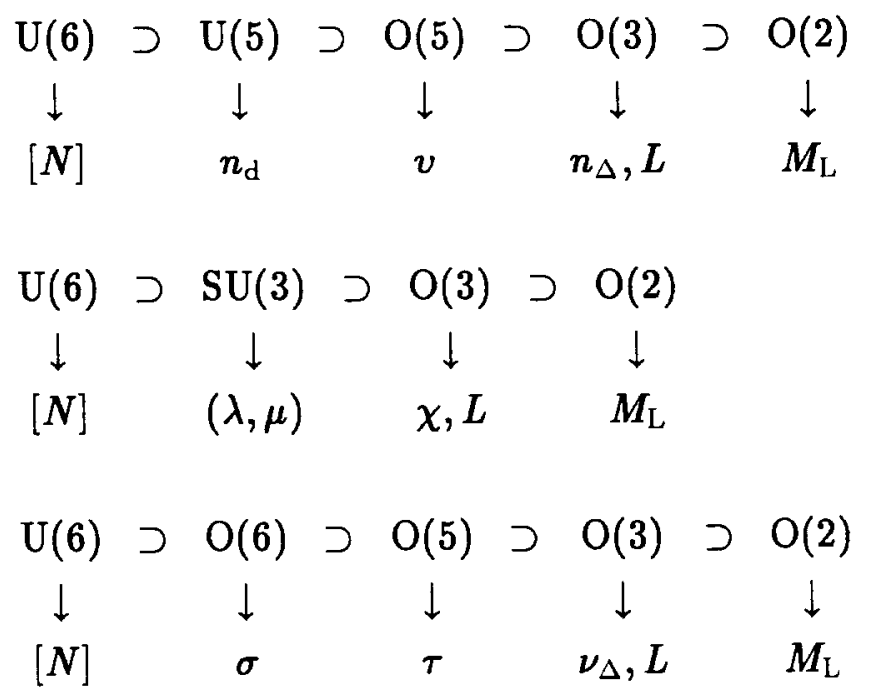

In principle, one may add Casimir operators of $U(6)$ to the Hamiltonians in (3.2). For a given nucleus they would reduce to a constant and hence they can be omitted if one is only interested in the description of the spectrum of a single nucleus. More significantly, none of the Hamiltonians contains a Casimir operator of $O(2)$. The occurrence of such a term would break the $O(3)$ symmetry (lift the $M_{\mathrm{L}}$ degeneracy) and would only be appropriate if the nucleus is placed in an external electric or magnetic field.

The properties of the dynamic symmetries (3.1) have been discussed extensively by Iachello and Arima (1987) and their relevance in the interpretation of nuclear structure 
data has been reviewed by Casten and Warner (1988) and Warner (1991). Given these thorough discussions of both theoretical and experimental aspects of the IBM-1, we refer the interested reader to them for details of the successes and limitations of the basic model. By now, the basic features of the predicted structures are familiar landmarks of nuclear structure, with the terms $\mathrm{U}(5), \mathrm{SU}(3)$ and $\mathrm{O}(6)$ being synonymous with their geometrical counterparts of vibrational, rotational and $\gamma$ unstable. Interest in the anharmonic vibrational structure incorporated in the $\mathrm{U}(5)$ symmetry has focused recently on the possible evidence for multiphonon excitations (Aprahamian et al., 1993, Délèze et al., 1993) while the $O(6)$ symmetry still stands out as an excellent example of the value and power of the algebraic approach. It emerged from the formalism as the third dynamical symmetry chain linking $\mathrm{U}(6)$ with $\mathrm{O}(3)$; i.e. its origins were purely algebraic, although the structure was later found to resemble that of the Wilets-Jean model of a $\gamma$-unstable rotor (Wilets and Jean, 1956). Its predictions were found to correspond closely to the empirical structure of some Pt nuclei (Cizewski et al., 1978) and indeed, the transition between $\mathrm{SU}(3)$ and $\mathrm{O}(6)$ structure was found to provide a natural explanation for the entire region of Os-Pt nuclei (Casten and Cizewski, 1978), which had always been regarded as one of the difficult challenges in nuclear structure. Most importantly, however, since that early work, it has become increasingly evident that the $O(6)$ symmetry in fact represents the third commonly occurring class of structure in nuclei. It has now been identified in many regions, most notably around $A=130$ (Casten et al., 1985). The experimental signatures which distinguish $\gamma$-soft and $\gamma$-rigid structures have been discussed by Zamfir and Casten (1991) who conclude that the experimental evidence indeed favours the former.

It is the algebraic aspect that we wish to highlight in this review and, since the majority of the work exploring the basic features of the IBM was done some years ago, we will not reiterate it here. The many current studies which utilise the IBM do so simply as the model of choice to treat a particular nucleus, or are directed at exploring specific extensions of the basic model to broaden its applicability far beyond the region where it was originally intended to apply, namely, the low-energy, low-spin collective excitations of nuclei. Thus such extensions might include the incorporation of $L=3$ or 4 bosons, configuration mixing with two-quasi-particle states or the inclusion of cubic terms in the Hamiltonian to induce triaxiality. To cite some specific examples, a recent study by Balantekin et al (1992) explores the effect of including $L=4$ bosons in the IBM basis on the predictions of the model for sub-barrier fusion probabilities. One can also obtain a good description of these features with a purely geometrical model (Leigh 
et al., 1993). Energy staggering in negative-parity bands spanning both vibrational and deformed nuclei has been studied (Casten et al., 1993) by incorporating an octupole boson in the Hamiltonian. However, while of great interest, these types of study tend to rely more on the numerical aspects of the calculations rather than the algebraic properties of the model and we therefore prefer to move on to discuss perhaps the most obvious and major extension to the original approach, which is to treat the neutron and proton degrees of freedom separately.

\section{Neutron-proton systems}

Up to this point the discussion has been couched in terms of bosons, and hence nucleons, of a single type. This is equivalent to the many macroscopic approaches to nuclear structure in which collective properties are described in terms of a single set of geometrical variables with no distinction being made between the neutron and proton degrees of freedom. However, such a distinction can be crucial in the description of particular nuclear properties, and even more so if the collective basis is eventually to be linked to the underlying single-particle shell structure.

The extension of the IBM to incorporate both neutrons and protons, referred to as IBM-2, was proposed by Arima et al. (1977). Its group structure can be summarised as

$$
\mathrm{U}_{\nu}(6) \otimes \mathrm{U}_{\pi}(6) \supset \mathrm{U}_{\nu \pi}(6) \supset\left\{\begin{array}{c}
\mathrm{U}_{\nu \pi}(5) \supset \mathrm{O}_{\nu \pi}(5) \\
\mathrm{SU}_{\nu \pi}(3) \\
\mathrm{O}_{\nu \pi}(6) \supset \mathrm{O}_{\nu \pi}(5)
\end{array}\right\} \supset \mathrm{O}_{\nu \pi}(3) \supset \mathrm{O}_{\nu \pi}(2)
$$

and starts from the product of the $\mathrm{U}(6)$ groups for neutrons and protons. This simply includes the two sets of generators representing the $s$ and $d$ degrees of freedom for each type of nucleon. It is then necessary to couple the neutron and proton degrees of freedom. This can, in principle, be done at any point in the identical decompositions of the two $\mathrm{U}(6)$ groups and gives rise to the sum groups whose generators have the form $b_{\nu}^{\dagger} \tilde{b}_{\nu}+b_{\pi}^{\dagger} \tilde{b}_{\pi}$. In physical terms, however, the point at which the two chains are coupled reflects the strength of the coupling between the two constituents. Coupling at the level of $\mathrm{O}(3)$, for instance, would represent a system in which the neutrons and protons each maintained their individual collective quantum numbers and only their respective angular momenta were eventually coupled to give the total $L$, represented by $\mathrm{O}_{\nu \pi}(3)$. In practice, however, the opposite is true, since the neutron-proton interaction is known to be dominant in determining collective properties. Hence the coupling is introduced at the highest point in 
the chains and gives rise to the group $\mathrm{U}_{\nu \pi}(6)$. The subsequent three dynamical symmetry chains are then identical to those of IBM-1, with the proviso that they involve the sum groups at each stage.

The generators of the sum groups are symmetric under interchange of $\nu$ and $\pi$, as are the operators which characterise the subgroups. Hence the structure arising from the three chains is identical to that of IBM-1; the only new feature is the group $U_{\nu \pi}(6)$. This group describes the symmetry of coupling the orbital motion of the valence neutrons and protons and essentially contains the 'new physics' offered by IBM-2. It leads to a new quantum number, $F$ spin, which distinguishes the fully symmetric states (equivalent to those of IBM-1) from those of lesser or 'mixed' symmetry (sometimes also referred as non-symmetric states). It is this latter class of states which represent modes of collective motion outside the scope of the one-fluid picture since they involve out-of-phase motion of neutrons versus protons.

In the reduction (4.1), $\mathrm{U}_{\nu \pi}(6)$ is characterised by the irreducible representations $[N-$ $f, f]$ where $N=N_{\nu}+N_{\pi}$ and

$$
f=0,1,2, \ldots, \min \left(N_{\nu}, N_{\pi}\right)
$$

The lowest states of the system are then contained in the representation $[N, 0]$ which denotes the totally symmetric coupling. These correspond to the states of the IBM-1. The next representation, $[N-1,1]$, and all succeeding ones, represent couplings of the neutron and proton $\mathrm{U}(6)$ structures which are not fully symmetric and therefore contain states outside the IBM-1 space. The quantum number $F$ is related to the label $f$ of (4.2) by

$$
F=\frac{1}{2} N-f \quad F_{z}=\frac{1}{2}\left(N_{\pi}-N_{\nu}\right)
$$

Hence,

$$
F_{\max }=\frac{1}{2}\left(N_{\nu}+N_{\pi}\right) \quad F_{\min }=F_{z} .
$$

As a simple example, consider a system with two bosons. In IBM-1 the lowest energy $2^{+}$ state is given by

$$
\left|2_{1}^{\dagger}\right\rangle=s^{\dagger} d^{\dagger}|0\rangle
$$

In the neutron-proton system two such states exist, viz.

$$
\left|2^{+}\right\rangle_{\mathrm{S}}=\sqrt{\frac{1}{2}}\left(s_{\pi}^{\dagger} d_{\nu}^{\dagger}+s_{\nu}^{\dagger} d_{\pi}^{\dagger}\right)|0\rangle \quad\left|2^{+}\right\rangle_{\mathrm{A}}=\sqrt{\frac{1}{2}}\left(s_{\pi}^{\dagger} d_{\nu}^{\dagger}-s_{\nu}^{\dagger} d_{\pi}^{\dagger}\right)|\mathrm{o}\rangle .
$$

The first is symmetric with respect to interchanging the $\nu$ and $\pi$ labels and the second antisymmetric. 


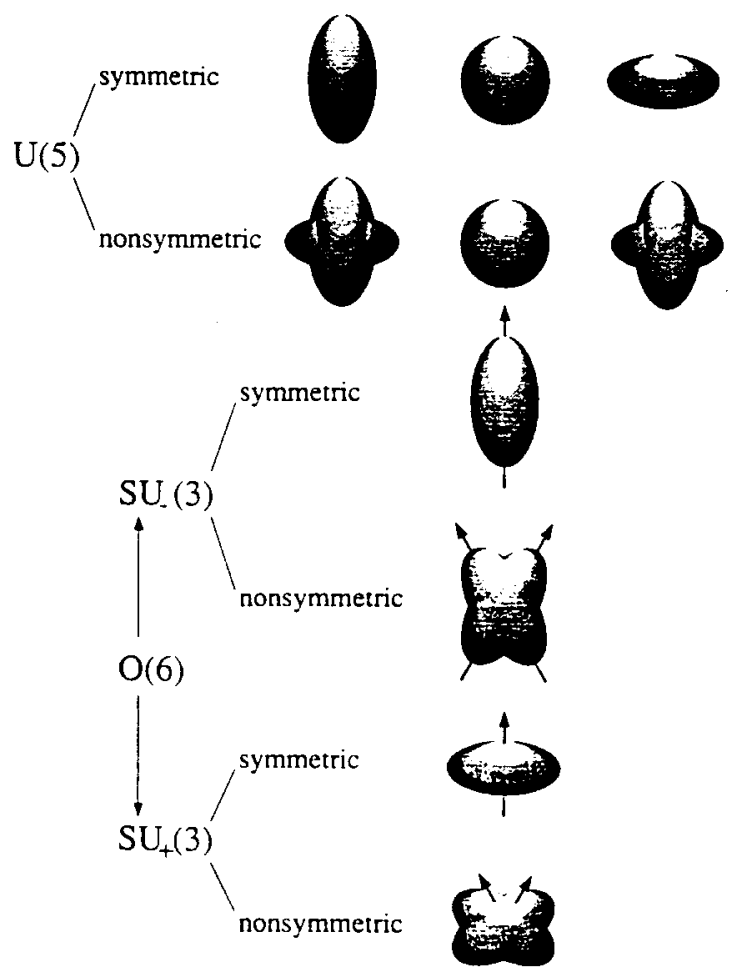

Figure 4.1: Schematic illustration of the geometrical shapes associated with symmetric and non-symmetric states of the IBM-2 (Van Isacker et al., 1986).

The analogy with isospin is immediately evident. However, here the exchange symmetry involves the bosons and hence the relative contributions of the neutron and proton orbital angular momenta to the total collective angular momentum. It does not imply an exchange symmetry at the level of the single-particle states, as in the case of isospin. Thus in heavier nuclei, where neutrons and protons occupy different major shells, the first states of non-maximal $F$ spin occur around $3 \mathrm{MeV}$ while the first states of non-minimal $T$ spin appear around $15 \mathrm{MeV}$.

Since the states of mixed symmetry are characterised by out-of-phase coupling between neutrons and protons, the relevant experimental probe is the study of M1 properties. A schematic illustration of the type of modes under discussion is given in figure 4.1. Details of the structure, quantum numbers and properties of the lowest non-symmetric states can be found in more comprehensive reviews (Van Isacker et al., 1986, Lipas et al., 1990). It is sufficient here to note that the 'normal' quadrupole vibrational modes each give rise to counterparts in which the neutrons and protons move against each other, rather than in phase. An additional possibility arises in deformed nuclei, where an oscillation in the angle between the deformed neutron and proton mass distributions leads 
to a collective band with $K^{\pi}=1^{+}$which represents the lowest mixed-symmetry mode in this class of nuclei. This is frequently referred to as the 'scissors' mode and is found at an excitation energy of about $3 \mathrm{MeV}$ in the rare-earth region with an M1 excitation strength of $1-3 \mu_{\mathrm{N}}^{2}$.

A large collective $B(\mathrm{M} 1)$ strength is, in fact, a signature of the mixed-symmetry modes in general, albeit sometimes of neccessity between $2^{+}$states. In lowest order, M1 transitions in IBM-1 are forbidden and the $g$ factors of all states are equal. These features are simply a consequence of the M1 operator being proportional to the total angular momentum and are common to any one-fluid model. In IBM-2 any transition operator has a neutron and a proton part, viz.

$$
T=t_{\nu} T_{\nu}+t_{\pi} T_{\pi}
$$

where $t_{\nu(\pi)}$ are the boson effective $g$ factors or charges. If the structures of $T_{\nu(\pi)}$ are identical and if $t_{\nu}$ and $t_{\pi}$ are equal, then $T$ is symmetric under the exchange of $\nu$ and $\pi$ and becomes a generator of $\mathrm{U}_{\nu \pi}(6)$. It thus cannot connect different representations of $\mathrm{U}_{\nu \pi}(6)$, i.e. states of different $F$ spin. In the case of the M1 operator, however, the $t_{\nu(\pi)}$ needed are the boson $g$ factors, where $g_{\nu} \approx 0 \mu_{\mathrm{N}}$ and $g_{\pi} \approx 1 \mu_{\mathrm{N}}$. The formalism then predicts strong M1 transitions between the lowest-lying fully-symmetric states with $F=F_{\max }$ and the first states of mixed symmetry with $F=F_{\max }-1$. M1 transitions between states of maximal $F$ spin still vanish.

In deformed nuclei the lowest mixed-symmetry $1^{+}$mode can be excited directly from the ground state via an M1 transition whose strength is given by (Scholten et al., 1985)

$$
B\left(\mathrm{M} 1 ; 0_{\mathrm{g}}^{+} \rightarrow 1_{\mathrm{M}}^{+}\right)=\frac{3}{4 \pi}\left(g_{\nu}-g_{\pi}\right)^{2} \frac{8}{2 N-1} N_{\nu} N_{\pi} .
$$

For the rare-earth region, and with $g_{\nu}=0 \mu_{N}$ and $g_{\pi}=1 \mu_{N}$, the above equation gives M1 strengths of 3-4 $\mu_{N}^{2}$. These values are reduced by roughly a factor of two if boson $g$ factors deduced from the systematics of $g\left(2_{1}^{+}\right)$values are used (Wolf et al., 1985).

The first experimental evidence for these collective $1^{+}$states came from electron scattering studies in ${ }^{156} \mathrm{Gd}$ (Bohle et al., 1984) which have subsequently led to an extensive series of measurements with electrons, protons and photons to characterise the location, nature and fragmentation of the scissors mode in deformed nuclei (Richter, 1991, Kneissl, 1992). A portion of the (e,e') spectrum in which the $1^{+}$state was first observed is shown in figure 4.2 along with the corresponding $\left(\gamma, \gamma^{\prime}\right)$ and $\left(\mathrm{p}, \mathrm{p}^{\prime}\right)$ spectra.

The nuclear resonance fluorescence (NRF) data provides the resolution to reveal five weakly excited states in the vicinity of the dominant peak, while the $\left(p, p^{\prime}\right)$ data gives 


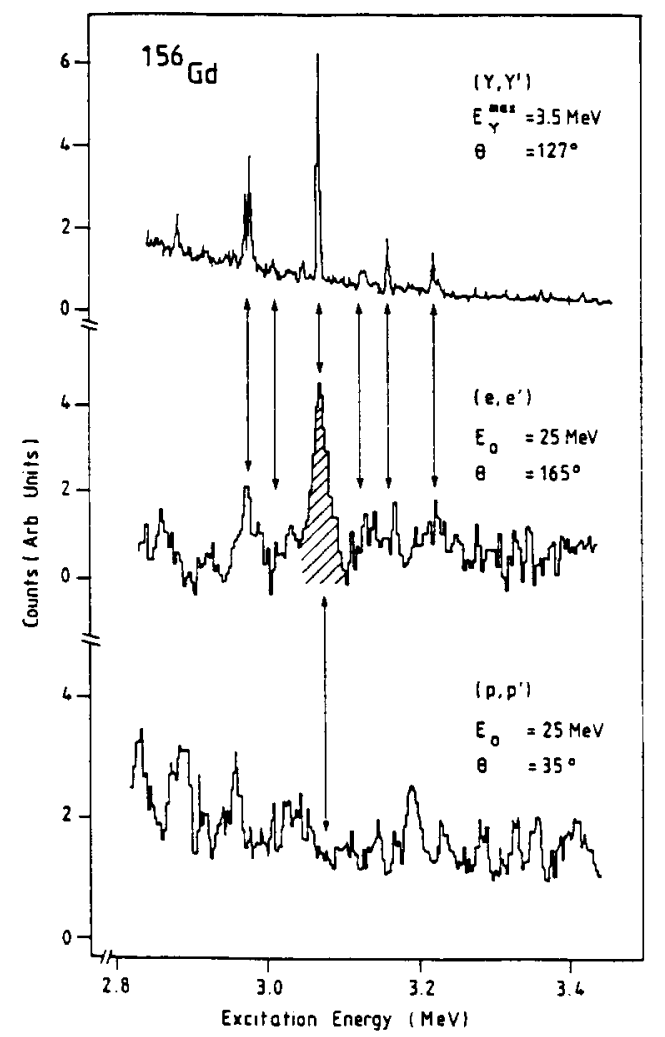

Figure 4.2: High-resolution nuclear resonance fluorescence and inelastic electron scattering spectra of ${ }^{156} \mathrm{Gd}$ (Richter, 1988). The arrows mark the $1^{+}$states.

empirical evidence of the dominantly orbital character of the excitation. Many additional studies have confirmed this latter characteristic, and have found a similar fragmentation of strength throughout the rare-earth and actinide nuclei.

In the IBM-2 the energy of the non-symmetric states is determined by the strength of the Casimir operator of $\mathrm{U}_{\nu \pi}(6)$. This is usually expressed in terms of a Majorana interaction of the form

$$
\xi_{2}\left(s_{\nu}^{\dagger} d_{\pi}^{\dagger}-d_{\nu}^{\dagger} s_{\pi}^{\dagger}\right)^{(2)} \cdot\left(\tilde{s}_{\nu} \tilde{d}_{\pi}-\tilde{d}_{\nu} \tilde{s}_{\pi}\right)^{(2)}-2 \sum_{k=1,3} \xi_{k}\left(d_{\nu}^{\dagger} d_{\pi}^{\dagger}\right)^{(k)} \cdot\left(\tilde{d}_{\nu} \tilde{d}_{\pi}\right)^{(k)}
$$

where, for simplicity, often $\xi_{1}=\xi_{2}=\xi_{3} \equiv \lambda$ is taken. The parameter $\lambda$ is thus a measure of the collective symmetry energy and its value has been found to be described by the empirical expression $\lambda / \delta=(3.4 \pm 0.8) /\left(N_{\nu} N_{\pi}\right)^{1 / 2} \mathrm{MeV}$ (Hartmann et al., 1987). An explicit dependence between the orbital M1 strength and deformation $\delta$ has been found as a result of a series of NRF studies of Sm isotopes (Ziegler et al., 1990). In this case, the relationship is $B(M 1) \propto \delta^{2}$, as illustrated in figure 4.3.

The lowest mixed-symmetry states in $\mathrm{U}(5)$ and $\mathrm{O}(6)$ type nuclei have $L^{\pi}=2^{+}$and hence the collective $B(M 1)$ strength in these cases is to the first-excited $2^{+}$state. Experimental evidence for these modes has also been found (Hamilton et al., 1984) although 


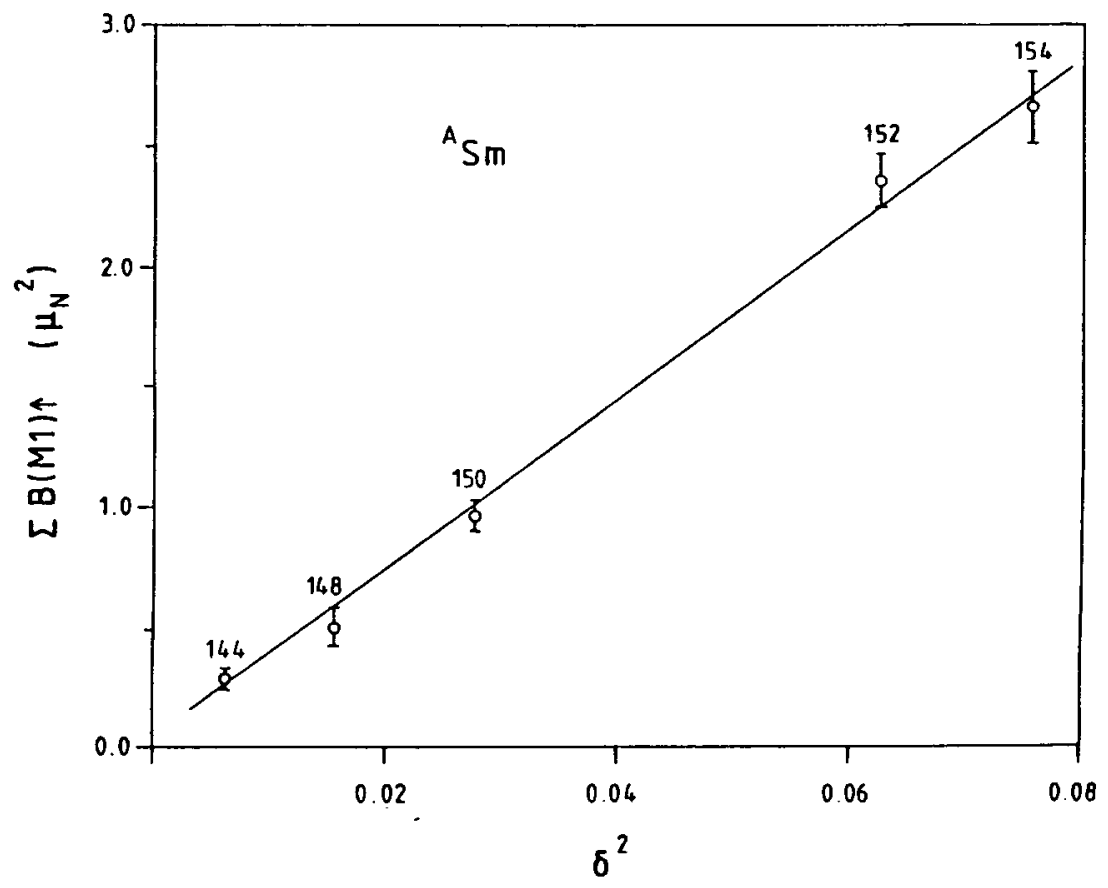

Figure 4.3: Orbital M1 strength versus $\delta^{2}$ (Ziegler et al., 1990).

here the results are far less extensive since the states cannot be excited by M1 transitions directly from the ground state.

The wealth of data which has now emerged confirming the existence of mixed-symmetry states has led to a concomitant effort to understand their microscopic origins. In particular, calculations within the framework of the random-phase approximation (RPA) have been performed by several groups (Faessler et al., 1990, Hamamoto and Åberg, 1984, 1986, Sugarawa-Tanabe and Arima, 1988, 1989, Zawischa and Speth, 1991) as have TDA calculations (De Coster and Heyde, 1991). Very recently, there have been studies (De Coster and Heyde, 1993, Ikeda and Shimano, 1993) investigating the role played by the centrifugal term in the deformed Hamiltonian in concentrating magnetic dipole strength in the low-lying $1^{+}$states in question. While these results and their differing conclusions concerning the structure and degree of collectivity of the $1^{+}$states are of great interest, they are not strictly the domain of the IBM and the algebraic approach. Indeed, they would warrant a review in their own right. Nevertheless it is worth noting that the first experimental results probing this question have now been obtained (Freeman et al., 1989, 1993a).

The study centred on the ${ }^{165} \mathrm{Ho}(\mathrm{t}, \alpha){ }^{164} \mathrm{Dy}$ reaction. The ground state of ${ }^{165} \mathrm{Ho}$ is the $7 / 2^{-}[523]$ Nilsson proton orbital which has dominantly $h_{11 / 2}$ parentage. The aim of the 


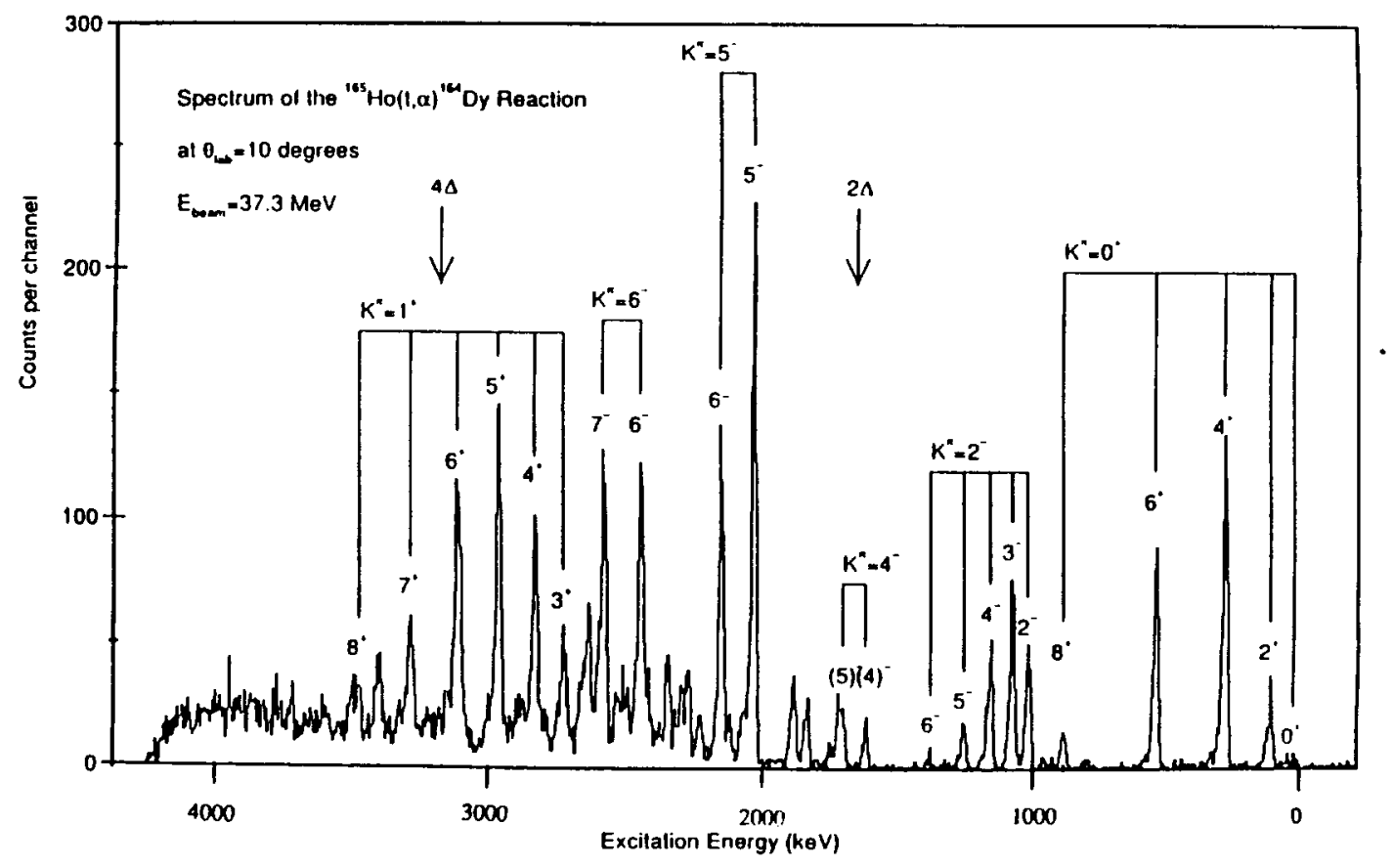

Figure 4.4: Position spectrum of $\alpha$ particles from the ${ }^{165} \mathrm{Ho}(\mathrm{t}, \alpha){ }^{164} \mathrm{Dy}$ reaction (Freeman et al., 1989).

experiment was to identify $l=5$ transfer into one or more of the $K^{\pi}=1^{+}$bands in ${ }^{164} \mathrm{Dy}$, whose bandheads had previously been identified from electron scattering (Bohle et al., 1987 ) and NRF work (Wesselborg et al., 1988). Clearly, in this region, contributions from $\left(h_{11 / 2}\right)^{2}$ two-quasi-proton configurations might be expected to constitute a major part of the total observed M1 strength. The results are shown in figure 4.4 and are surprising for a number of reasons. An $l=5 K^{\pi}=1^{+}$band was clearly identified at $2.557(15) \mathrm{MeV}$ with the $\left(h_{11 / 2}\right)^{2}$ component in the intrinsic wavefunction estimated to be $\sim 90 \%$. The results of the NRF experiments show two groups of $1^{+}$states, centred around 2.5 and $3.1 \mathrm{MeV}$, carrying total M1 strengths of 1.67 and $3.15 \mu_{\mathrm{N}}^{2}$, respectively. The upper group is less fragmented and corresponds in energy and strength to the expected behaviour of the scissors mode; the transfer data show no population in this energy region, consistent with a collective state. In the lower group there are two states which could correspond to the $2.557(15) \mathrm{MeV}$ band seen in the transfer data. However, their M1 strengths are only 0.30 and $0.48 \mu_{\mathrm{N}}^{2}$. Hence the majority of M1 strength in this nucleus remains unexplained and the single-particle structure of the M1 mode is still an open question. A similar investigation of the two-quasiparticle structure in ${ }^{174} \mathrm{Yb}$ failed to populate any $\mathrm{K}^{\pi}=1^{+}$ bands (Freeman et al., 1993b)

There are other aspects of mixed-symmetry states which pose intriguing questions. For instance, the structures discussed so far involve only the lowest possible neutron- 


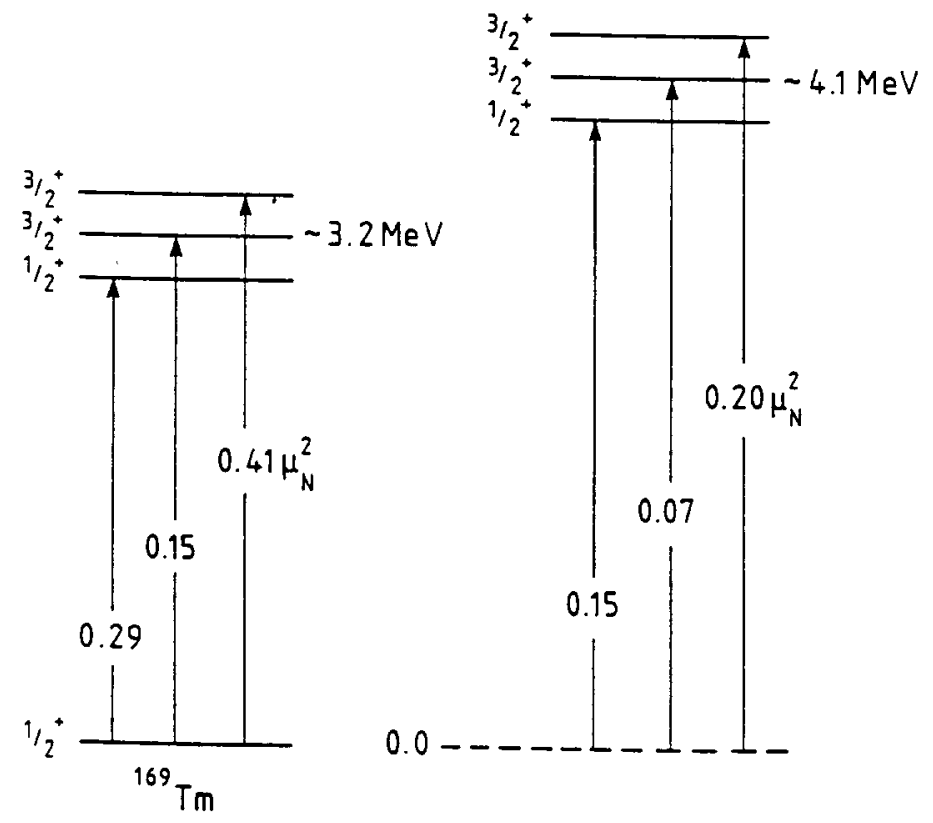

Figure 4.5: Predicted M1 strengths in ${ }^{169} \mathrm{Tm}$ (Van Isacker and Frank, 1989).

proton modes. There are higher-lying excitations predicted which have yet to be identified, such as the mixed-symmetry counterparts of the $\beta$ and $\gamma$ vibrations in deformed nuclei. These would be characterised by enhanced M1 transitions to the symmetric $\beta$ and $\gamma$ bands. Also, the predictions have been extended to encompass odd-mass nuclei (Van Isacker and Frank, 1989, Frank et al., 1991) and the result for ${ }^{169} \mathrm{Tm}$ is shown in figure 4.5. Recently a concentration of dipole strength near $3 \mathrm{MeV}$ was found in a nuclear resonance fluorescence experiment on the deformed nucleus ${ }^{163} \mathrm{Dy}$ (Bauske et al., 1993). Although spin and parity could not be established experimentally, energy systematics of M1 excitations in neighbouring even-even Dy isotopes suggest the corresponding levels in ${ }^{163} \mathrm{Dy}$ have a scissors character. It is as yet not clear whether these enhanced M1 excitations are as common a phenomenon in odd-mass nuclei as they are in even-even isotopes. The odd-mass case deserves further study though since it can shed light on the band structure of mixed-symmetry states and thereby perhaps once and for all settle the question of the collectivity of these states.

The concept of $F$ spin can also be used to understand M1 transitions between lowlying collective states. The existence of such transitions has long been known and various approaches have been tried in the past to incorporate them within a collective framework. In IBM-2 they arise naturally through the small admixtures of the higher-lying mixedsymmetry states which occur with realistic Hamiltonians. Indeed, despite the goodness of the $F$-spin quantum number, it would be surprising if such mixing did not exist to some 
extent, and the $\mathrm{M} 1$ properties serve as a direct probe of its magnitude.

An $F$-spin mixing is generated by the presence of a neutron-proton asymmetry in the Hamiltonian. For the common case of a Hamiltonian dominated by a $Q \cdot Q$ interaction, one has in general three possible terms of the form

$$
\kappa_{\nu} Q_{\nu} \cdot Q_{\nu}+\kappa_{\pi} Q_{\pi} \cdot Q_{\pi}+\kappa_{\nu \pi} Q_{\nu} \cdot Q_{\pi}
$$

An $F$-spin symmetric Hamiltonian requires a quadrupole interaction of the form $Q \cdot Q$ with $Q=Q_{\nu}+Q_{\pi}$ and is obtained for $\kappa_{\nu}=\kappa_{\pi} \equiv \bar{\kappa}$ and $\kappa_{\nu \pi}=2 \bar{\kappa}$. An additional condition for $F$-spin symmetry, is that the quadrupole operators

$$
Q_{\rho}=\left(s_{\rho}^{\dagger} \tilde{d}_{\rho}+d_{\rho}^{\dagger} \tilde{s}_{\rho}\right)^{(2)}+\chi_{\rho}\left(d_{\rho}^{\dagger} \tilde{d}_{\rho}\right)^{(2)} \quad \rho=\nu, \pi
$$

must have $\chi_{\nu}=\chi_{\pi}$. Thus two mechanisms for inducing $F$-spin mixing in the Hamiltonian are immediately apparent: (i) via $\Delta \kappa$ breaking, i.e. $\Delta \kappa=\kappa_{\nu \pi}-2 \bar{\kappa} \neq 0$ and (ii) via $\Delta \chi$ breaking, i.e. $\Delta \chi=\left(\chi_{\nu}-\chi_{\pi}\right) / 2 \neq 0$.

The effect of these symmetry-breaking terms was first studied in a perturbation approach (Dieperink et al., 1987) where it was demonstrated that the $\Delta \chi$ breaking produces the dominant effect on $\mathrm{M} 1$ properties. A number of subsequent detailed numerical studies (Harter et al., 1986, Van Isacker et al., 1988) have confirmed this and, while differing somewhat in the approaches used, have also shown that the value of $\Delta \chi$ is almost solely determined by the M1 strengths, i.e. it is largely independent of energies and $B(\mathrm{E} 2)$ values which are instead determined by $\epsilon_{\mathrm{d}} / \bar{\kappa}$, the competition between the spherical and deformation-driving terms, and the sum of $\chi_{\nu}$ and $\chi_{\pi}$. Moreover, the relative magnitudes of $\chi_{\nu}$ and $\chi_{\pi}$ were shown to uniquely determine the sign of $\delta(\mathrm{E} 2 / \mathrm{M} 1)$ mixing ratios between the $\gamma$ and ground bands in deformed nuclei. Since this sign is known to be negative in the rare-earth nuclei, it leads to the constraint $\chi_{\nu}>\chi_{\pi}$. This, in turn, predicts that $g\left(2_{\gamma}^{+}\right) / g\left(2_{\mathrm{g}}^{+}\right) \approx 0.85$, a ratio which seems consistent with existing data. Examples of some of the predictions for $\mathrm{M} 1$ properties are given in tables 1 and 2.

Thus the algebraic approach in this case has identified the specific new physics which enters the problem when neutrons are distinguished from protons and has defined a new quantum number to label the resulting exchange symmetry. Moreover, a corollary of the preceeding discussion is that quadrupole properties alone will not uniquely determine the parameters of an IBM-2 Hamiltonian. Energies and E2 properties could be described by an IBM-1 Hamiltonian which can, in principle, be projected from an infinite number of IBM-2 Hamiltonians. To provide an unambiguous determination of the parameters of 
Table 4.1: Reduced M1 matrix elements for $\gamma \rightarrow g$ and $\gamma \rightarrow \gamma$ transitions in ${ }^{168} \mathrm{Er}$

\begin{tabular}{llll}
\hline \hline & & \multicolumn{2}{c}{$\mid\left\langle L_{\mathrm{f}}\|T(\mathrm{M} 1)\| L_{\mathrm{i}}\right\rangle\left(\mu_{\mathrm{N}}\right)$} \\
\cline { 4 - 4 }$L_{\mathrm{i}}$ & $L_{\mathrm{f}}$ & Experiment & Theory \\
\hline $2_{\gamma}$ & $2_{\mathrm{g}}$ & & 0.029 \\
$3_{\gamma}$ & $2_{\mathrm{g}}$ & $0.070(15)$ & 0.038 \\
$3_{\gamma}$ & $4_{\mathrm{g}}$ & $0.049(3)$ & 0.041 \\
$4_{\gamma}$ & $4_{\mathrm{g}}$ & & 0.079 \\
$5_{\gamma}$ & $4_{\mathrm{g}}$ & $0.08(5)$ & 0.072 \\
$6_{\gamma}$ & $6_{\mathrm{g}}$ & $0.014(6)$ & 0.13 \\
$3_{\gamma}$ & $2_{\gamma}$ & $0.160(5)$ & 0.16 \\
$4_{\gamma}$ & $3_{\gamma}$ & & 0.21 \\
$5_{\gamma}$ & $4_{\gamma}$ & $0.23(1)$ & 0.24 \\
$6_{\gamma}$ & $5_{\gamma}$ & $0.20(4)$ & 0.27 \\
$7_{\gamma}$ & $6_{\gamma}$ & $0.27(6)$ & 0.29 \\
\hline \hline
\end{tabular}

Table 4.2: Reduced M1 matrix elements for $\gamma \rightarrow g$ and $\gamma \rightarrow \gamma$ transitions in ${ }^{184} \mathrm{~W}$

\begin{tabular}{llll}
\hline \hline & & \multicolumn{2}{l}{$\left|\left\langle L_{\mathrm{f}}\|T(\mathrm{M} 1)\| L_{\mathrm{i}}\right\rangle\right|\left(\mu_{\mathrm{N}}\right)$} \\
\cline { 3 - 4 }$L_{\mathrm{i}}$ & $L_{\mathrm{f}}$ & Experiment & Theory \\
\hline $2_{\gamma}$ & $2_{\mathrm{g}}$ & $0.020(1)$ & 0.021 \\
$3_{\gamma}$ & $2_{\mathrm{g}}$ & $0.030(2)$ & 0.025 \\
$3_{\gamma}$ & $4_{\mathrm{g}}$ & $0.032(3)$ & 0.034 \\
$4_{\gamma}$ & $4_{\mathrm{g}}$ & $0.03(3)$ & 0.046 \\
$3_{\gamma}$ & $2_{\gamma}$ & & 0.068 \\
$4_{\gamma}$ & $3_{\gamma}$ & & 0.085 \\
\hline \hline
\end{tabular}


IBM-2, and hence test their underlying microscopic description, it is essential to use the M1 properties which explicitly probe the separation of the neutron and proton degrees of freedom.

\section{Odd-mass nuclei}

The extension of the general framework of the IBM to encompass the coupling of an odd particle is referred to as the Interacting Boson-Fermion Model (IBFM). The IBFM can be thought of, on one level, as simply a core-particle coupling model with the advantage of a rather versatile and extensive description of the collective core states, by virtue of the IBM Hamiltonian. However, there are two aspects of the approach which go further. Firstly, as for the even-even case, it is possible to forge a link between the collective Hamiltonian and the underlying single-particle shell structure. Secondly, and most importantly for this review, it is the existence and role of symmetries which lends the IBM its most unique and characteristic features, and such a comment is no less true for the IBFM. The three fundamental symmetries which emerge from the algebraic treatment of the boson problem reappear in the odd-mass formalism although, as will be seen, their existence and structure now depends critically on the single-particle space available to the odd fermion.

In the previous section we saw how the algebra of the neutron-proton problem involves the coupling of the two group structures in terms of product and sum groups. The basic approach is the same in the boson-fermion case. However, the algebraic structure of bilinear products of fermion operators, where $j$ can assume more than one value, is much richer than that for boson operators and hence the study of possible fermion algebras and their coupling to the boson degrees of freedom becomes much more complex than the problem of section 4 . No attempt will be made here to go into the details of such algebras, but the interested reader is referred to the recent texts of Iachello and Van Isacker (1991) and Warner (1993). Rather, we will simply use some of the concepts introduced earlier to show how boson-fermion symmetries arise and to discuss the constraints they imply on the overall form of the core-particle Hamiltonian.

The basic group structures associated with the IBFM are $\mathrm{U}^{\mathrm{B}}(6)$ and $\mathrm{U}^{\mathrm{F}}(m)$ where $m=\sum_{i}\left(2 j_{i}+1\right) ; \mathrm{U}^{\mathrm{B}}(6)$ is the usual boson group describing the collective excitations in the core Hamiltonian while $\mathrm{U}^{\mathrm{F}}(m)$ is the group of unitary transformations describing the single-particle space. The starting point for the boson-fermion problem is then the direct 
product group

$$
\mathrm{U}^{\mathrm{B}}(6) \otimes \mathrm{U}^{\mathrm{F}}(m)
$$

which contains the two sets of generators, boson and fermion. The next step is to consider how the boson and fermion groups can be coupled, and whether a chain of subgroups can be constructed which leads to one or more dynamical symmetries.

The basic approach to the coupling of two group structures and the definition of dynamical symmetries has already been discussed in the preceeding sections. Again, the basic physical requirement in the process is that any chain ends with the subgroup whose generators are those of the total angular momentum. In the boson-fermion case the angular momentum group is usually denoted as $\operatorname{Spin}^{\mathrm{BF}}(3)$, with Casimir operator $J^{2}$ where $J=L+j$, the sum of the boson and single-particle angular momenta. The symmetries which can now appear are, however, dependent on the constituents of $\mathrm{U}^{\mathrm{F}}(m)$, i.e. which orbits - and therefore $j$ values-are included. There are thus a large number of schemes which can be proposed. Here we will concentrate on a particular class of symmetries which give rise to a rich structure encompassing all three IBM limits, and which rely for their construction on a pseudo-spin decomposition.

It was pointed out many years ago (Hecht and Adler, 1969, Arima et al., 1969) that it is possible to transform a single-particle basis described in terms of angular momentum variables $l, s$ and $j$ to a basis involving pseudo-orbital and pseudo-spin variables $\tilde{l}$ and $\tilde{s}$. These new variables do not, in general, correspond to the real orbital and spin angular momenta, but the total $j$ is conserved. As an example, consider a set of orbits with $j=1 / 2,3 / 2,5 / 2,7 / 2$. In terms of pseudo variables this set could be described as either

$$
\tilde{l}=1,3 \quad \tilde{s}=1 / 2
$$

or

$$
\tilde{l}=2 \quad \tilde{s}=3 / 2 .
$$

Such a transformation does not, per se, change the physics of the problem but can prove crucial in constructing fermion algebras which reveal symmetries. In the case of $\tilde{s}=1 / 2$ the transformation is equivalent to the reduction

$$
\mathrm{U}^{\mathrm{F}}(m) \supset \mathrm{U}^{\mathrm{F}}(m / 2) \otimes \mathrm{SU}^{\mathrm{F}}(2)
$$

A symmetry of particular significance occurs whenever the reduction above leads to a set of $\tilde{l}$ values which correspond to a representation $\left(\lambda_{\mathrm{F}}, \mu_{\mathrm{F}}\right)$ of $\mathrm{SU}^{\mathrm{F}}(3)$. A necessary condition for this to happen is that the single-particle space must contain $j=1 / 2,3 / 2,5 / 2, \ldots, n+1 / 2$, 


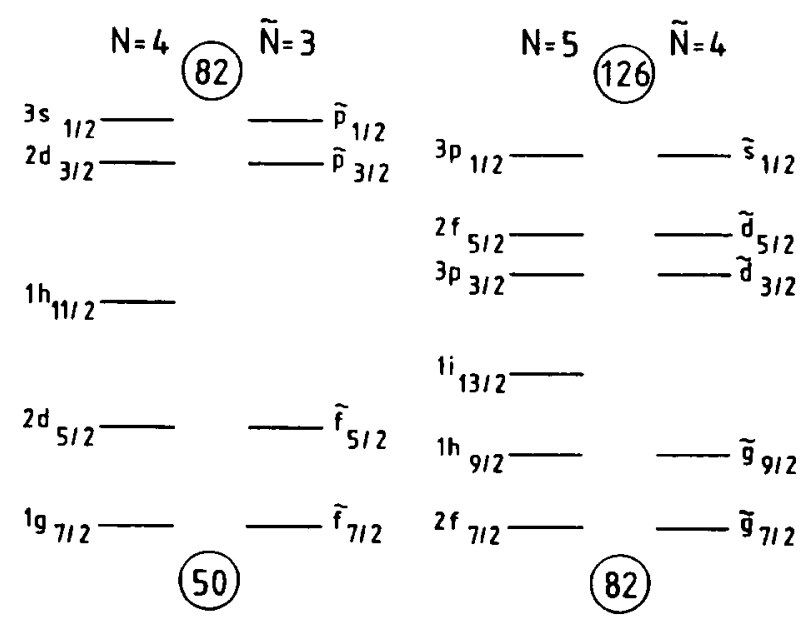

Figure 5.1: The effect of the pseudo-spin transformation for the $N=4$ and 5 shells. Pseudo quantum numbers are distinguished by a tilde.

to some maximum defined by $n$. The group $\mathrm{U}^{\mathrm{F}}(m / 2)$ then always contains $\mathrm{SU}^{\mathrm{F}}(3)$ as a subgroup so that the following dynamical symmetry can be formed

$$
\begin{aligned}
\mathrm{U}^{\mathrm{B}}(6) \otimes \mathrm{U}^{\mathrm{F}}(m) & \supset \mathrm{U}^{\mathrm{B}}(6) \otimes \mathrm{U}^{\mathrm{F}}(m / 2) \otimes \mathrm{SU}^{\mathrm{F}}(2) \\
& \supset \mathrm{SU}^{\mathrm{B}}(3) \otimes \mathrm{SU}^{\mathrm{F}}(3) \otimes \mathrm{SU}^{\mathrm{F}}(2) \\
& \supset \mathrm{SU}^{\mathrm{BF}}(3) \otimes \mathrm{SU}^{\mathrm{F}}(2) \\
& \supset \mathrm{O}^{\mathrm{BF}}(3) \otimes \mathrm{SU}^{\mathrm{F}}(2) \\
& \supset \mathrm{Spin}^{\mathrm{BF}}(3) .
\end{aligned}
$$

Note that the pseudo spin, represented by the group $\mathrm{SU}^{\mathrm{F}}(2)$, is only coupled at the final stage. Physically this means that the pseudo spin is decoupled from the problem and that the resulting Hamiltonian is independent of $\tilde{s}$, apart from the $J(J+1)$ splitting induced by the (diagonal) Casimir operator of $\operatorname{Spin}^{\mathrm{BF}}(3)$. This pseudo-spin symmetry is a crucial underlying assumption of these schemes and requires for its realisation a degeneracy in the single-particle space between orbits with $j=\tilde{l} \pm 1 / 2$ or 'pseudo-spin-orbit partners'. It is thus particularly fortunate that the shell model, by chance or by design, comes close to achieving this degeneracy. The effect of the transformation on the angular momentum variables for the $N=4$ and 5 shells is illustrated in figure 5.1. The set of normal-parity states in each case, excluding the state of largest $j$ for each $N$, now forms the complete set of states belonging to the pseudo-oscillator shell with $\tilde{N}=N-1$. Most importantly, the spin-orbit splitting is greatly reduced in the pseudo scheme. This property allows the 
fermion space to be treated in terms of $\mathrm{SU}(3)$ quantum numbers and leads to the pseudo $\mathrm{SU}(3)$ fermion model proposed by Ratna Raju et al. (1973) as well as to the associated pseudo-Nilsson scheme (Draayer, 1993).

In the last few years, the role played by pseudo-spin symmetry has attracted renewed interest. In particular, some of the analytic predictions associated with the quantum numbers of the pseudo-Nilsson model have been shown to be particularly relevant to high-spin studies, and the associated degeneracies have been shown to be the principal determinant of shell gaps in the deformed scheme, and hence of the location of regions of super(hyper)deformation (Dudek et al., 1987, Bahri et al., 1993). In addition, the initial discovery of identical superdeformed bands (i.e., superdefomed rotational bands with nearidentical moments of inertia) in neighbouring odd and even-even nuclei by Byrski et al. (1990) in the $A=150$ region has now been expanded to include numerous other examples in both the $A=150$ and 190 regions. A large number of examples of this phenomenon have also now been proposed among low-spin bands of normal deformation (Baktash et al., 1993b). It is difficult to reconcile these features with our existing understanding of pair correlations, which would predict a far larger change in the moment of inertia when an odd particle is added. In the original case, Nazarewicz et al. (1990) suggested that the required stability might occur if the decoupling parameter of the $\Omega=1 / 2$ orbital near the Fermi surface takes its pseudospin symmetry value of exactly \pm 1 . Stephens et al. (1990) have also used pseudospin symmetry to explain the near-integer alignments found in superdeformed bands in the $A=190$ region and have suggested that the quality of a pseudo $\mathrm{SU}(3)$ symmetry may be consistent with a compensation between deformation and alignment effects which acts to keep the moment of inertia constant. However, a recent discussion by Baktash et al. (1993a) of the many different models so far proposed to explain identical bands concludes that, as yet, no single scenario can adequately account for the occurrence of the phenomenon over such a broad range of nuclei and excitation regions.

Returning now to the boson-fermion $\mathrm{SU}(3)$ symmetry of (5.5), it is important to realise that, unlike the pseudo-Nilsson scheme, the boson-fermion approach involves the laboratory frame and hence incorporates the coupling of the core. The subgroup $\mathrm{O}^{\mathrm{BF}}(3)$ represents the pseudo-orbital plus core angular momentum of the system and gives rise to a new quantum number $\tilde{L}$, with $\tilde{L}=L+\tilde{l}$ and $J=\tilde{L} \pm 1 / 2, L$ being the core (or boson) angular momentum. In the limit of a pseudo-spin-independent Hamiltonian, $\tilde{L}$ will be a good quantum number, and this will be true for any symmetry scheme based on the 


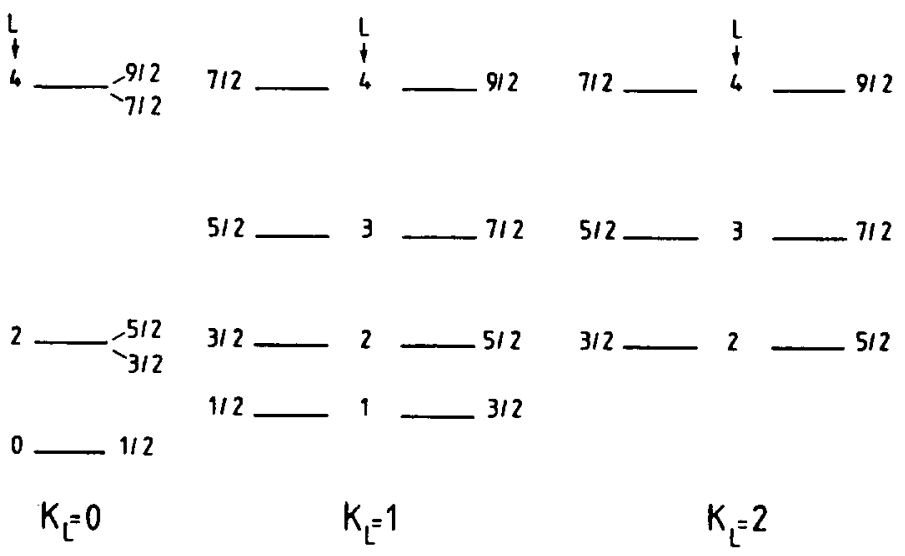

Figure 5.2: Rotational band structures in the pseudo-SU(3) scheme for $K_{\mathrm{L}}=0,1,2$. States are labelled by the quantum numbers $\tilde{L}$ and $J$.

pseudo-spin transformation. In $\mathrm{SU}(3)$, which describes axially-symmetric deformation, we can also define the projection of $\tilde{L}$ on the symmetry axis as $K_{\mathrm{L}}$ and it is clear that $K_{\mathrm{L}}$ will be equal to $\tilde{\Lambda}$, the pseudo-Nilsson quantum number describing the projection of $\tilde{l}$ on the symmetry axis. The structure of rotational bands which emerges in a boson-fermion $\mathrm{SU}(3)$ scheme of this type is shown schematically in figure 5.2. For each $K_{\mathrm{L}}$ value a series of $\tilde{L}$ values appears which corresponds to those which would be expected in an even-even nucleus with $K=K_{\mathrm{L}}$. Each $\tilde{L}$ value then gives rise to a couplet of states with $J=\tilde{L}_{ \pm 1 / 2}$. Thus the $K_{\mathrm{L}}=1$ case, for example, would describe a pair of bands with $K=1 / 2$ and $3 / 2$, while $K_{\mathrm{L}}=2$ yields a $K=3 / 2$ and a $5 / 2$ band. The states are now described by a basis $\left|K_{\mathrm{L}} \tilde{L} J M\right\rangle$, rather than a good- $K$ basis $\left|K_{\mathrm{L}} K J M\right\rangle$, and the scheme is likely to be valid in the limit of large pseudo-Coriolis coupling (Bohr et al., 1982, Frank et al., 1986) between the two bands which stem from a given $K_{\mathrm{L}}$ structure.

The example of the deformed single-particle scheme of figure 5.3 is familiar to everyone, as are the Nilsson quantum numbers $\left[N n_{3} \Lambda\right] \Omega$ labelling each deformed orbit. In the IBFM it has been shown (Bijker and Kota, 1988) that an equivalent result can always be obtained within an $\mathrm{SU}(3)$ coupling scheme. The resulting quantum numbers $(\lambda, \mu) K_{\mathrm{L}}$ are shown on the right of the figure. In the pseudo-Nilsson scheme the orbits shown form the complete set of states belonging to the $\tilde{N}=4$ shell and have quantum numbers $\left[\tilde{N} \tilde{n}_{3} \tilde{\Lambda}\right] \Omega$ where $\tilde{N}=N-1, \tilde{n}_{3}=n_{3}, \tilde{\Lambda}=\Lambda \pm 1$ and $\tilde{\Omega}=\tilde{\Lambda} \pm 1 / 2$.

The new quantum numbers associated with figure 5.2 can be tested via analytic expressions and selection rules for observables. A specific example, ${ }^{185} \mathrm{~W}$, has been studied in some detail by Warner and Bruce (1984). A more general result has been obtained 


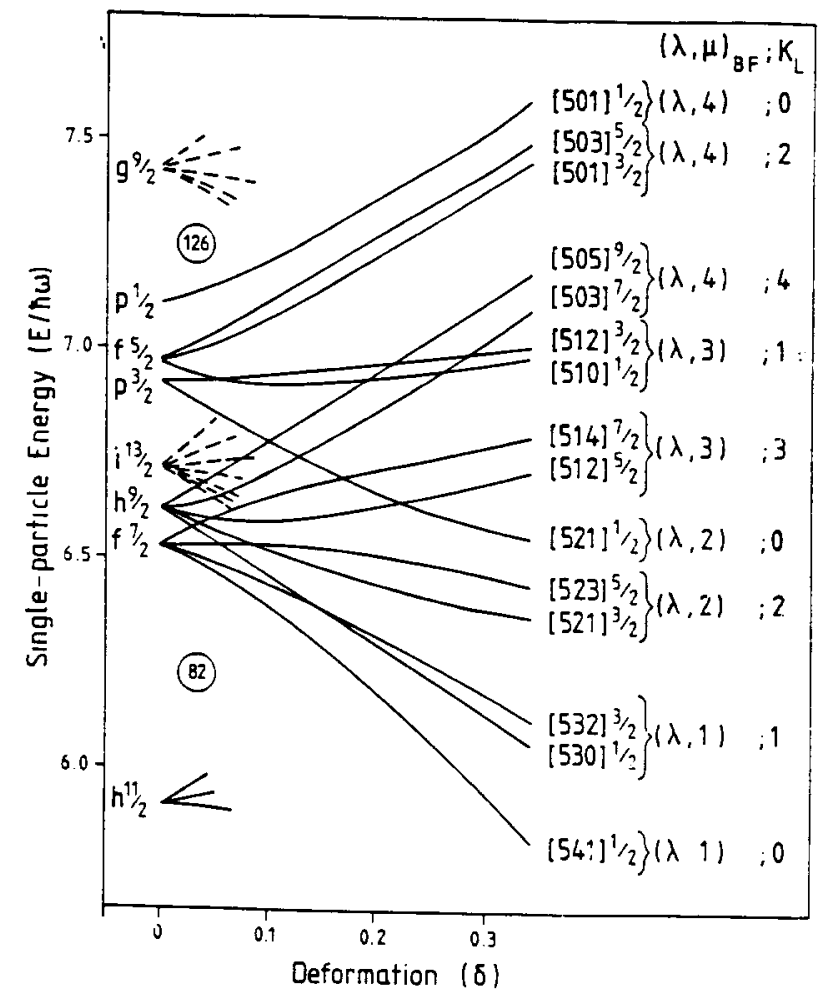

Figure 5.3: Nilsson $\left[N n_{3} \Lambda\right] \Omega$ and the $\mathrm{SU}(3)(\lambda, \mu)_{\mathrm{BF}} K_{\mathrm{L}}$ classification of deformed orbits originating from the 82-126 spherical shell (Bijker and Kota, 1988).

recently by Warner and Van Isacker (1990) concerning rotational intensity rules for E2 transitions, where the usual dependence on a single Clebsch-Gordan coefficient is replaced by

$$
\begin{aligned}
& B\left(\mathrm{E} 2 ; K_{\mathrm{L}_{\mathrm{i}}} \tilde{L}_{\mathrm{i}} J_{\mathrm{i}} \rightarrow K_{\mathrm{L}_{\mathrm{f}}} \tilde{L}_{\mathrm{f}} J_{\mathrm{f}}\right) \\
& =\frac{5}{16 \pi} e^{2} Q_{0}^{2}\left(2 J_{\mathrm{f}}+1\right)\left(2 \tilde{L}_{\mathrm{i}}+1\right)\left(\tilde{L}_{\mathrm{i}} K_{\mathrm{L}_{\mathrm{i}}} 2 K_{\mathrm{L}_{\mathrm{f}}}-K_{\mathrm{L}_{\mathrm{i}}} \mid \tilde{L}_{\mathrm{f}} K_{\mathrm{L}_{\mathrm{f}}}\right)^{2}\left\{\begin{array}{ccc}
\tilde{L}_{\mathrm{i}} & \tilde{L}_{\mathrm{f}} & 2 \\
J_{\mathrm{f}} & J_{\mathrm{i}} & 1 / 2
\end{array}\right\}^{2}
\end{aligned}
$$

where $Q_{0}$ is the intrinsic quadrupole moment. For $K_{\mathrm{L}}=0$ this expression yields identical results to the good- $K$ basis with $K=1 / 2$. However, for $K_{\mathrm{L}} \neq 0$ the results differ from a good- $K$ basis and, in some cases, the difference is substantial. This is apparent from table 3 where the two sets of predictions are compared with the available data for the low-lying $K=1 / 2$ and $3 / 2$ bands in ${ }^{187} \mathrm{Os}$, which correspond to the $K_{\mathrm{L}}=1$ case of figure 5.2 .

Of special interest is the appearance of interband transitions in the pseudo- $L$ scheme, of similar strength to the in-band transitions. This feature stems from the fact that the symmetry implicitly assumes $K$ mixing between the bands. The explicit form of the mixing is given by the transformation between the two bases, good- $\tilde{L}$ and good- $K$, which 
Table 5.1: Relative $B(\mathrm{E} 2)$ values in ${ }^{187} \mathrm{Os}$

\begin{tabular}{rrrrr}
\hline \hline & & \multicolumn{3}{c}{ Relative $B\left(\mathrm{E} 2 ; L_{\mathrm{i}} \rightarrow L_{\mathrm{f}}\right)$} \\
\cline { 3 - 5 }$K_{\mathrm{i}}, J_{\mathrm{i}}$ & $K_{\mathrm{f}}, J_{\mathrm{f}}$ & Experiment & $\tilde{L}$ basis & $K$ basis \\
\hline $1 / 2,5 / 2$ & $1 / 2,1 / 2$ & $100(9)$ & 100 & 100 \\
& $1 / 2,3 / 2$ & $135(50)$ & 86 & 29 \\
& $3 / 2,3 / 2$ & $41(21)$ & 29 & 0 \\
& $3 / 2,5 / 2$ & $24(12)$ & 21 & 0 \\
$3 / 2,5 / 2$ & $1 / 2,1 / 2$ & $65(7)$ & 29 & 0 \\
& $3 / 2,3 / 2$ & $100(37)$ & 100 & 100 \\
$3 / 2,7 / 2$ & $3 / 2,3 / 2$ & $100(12)$ & 100 & 100 \\
& $3 / 2,5 / 2$ & $122(66)$ & 75 & 150 \\
\hline \hline
\end{tabular}

takes the form (Van Isacker et al., 1987)

$$
\left|K_{\mathrm{L}} \tilde{L} J M\right\rangle=\left(\frac{2 \tilde{L}+1}{2 J+1}\right)^{1 / 2} \sum_{K_{\mathrm{s}} K}\left(\tilde{L} K_{\mathrm{L}} \tilde{s} K_{\mathrm{s}} \mid J K\right)\left|K_{\mathrm{L}} K J M\right\rangle
$$

Unfortunately, existing data are of insufficient accuracy to test the symmetry predictions conclusively. This is a common problem in odd-mass nuclei and calls for further experimental study. It would also be of considerable interest to explore the relevance of these predictions to high-spin states and, in particular, states in the second (superdeformed) minimum. Indeed, as mentioned earlier, the role of pseudo spin in the structure of these latter states is currently a topic of some interest. For large $J$ values, (5.6) indicates that, for $\Delta J=2$ transitions, the $B(\mathrm{E} 2)$ values approach the normal rotational rules; for $\Delta J=1$ transitions, however, the differences between the two descriptions are substantial and given by the limiting expressions (valid for large $J$ )

$$
\begin{aligned}
& K=K_{\mathrm{L}}-1 / 2: B(\mathrm{E} 2 ; J \rightarrow J-1)_{\mathrm{L}} \simeq\left(\frac{2 K+1}{2 K}\right)^{2} B(\mathrm{E} 2 ; J \rightarrow J-1)_{\mathrm{K}} \\
& K=K_{\mathrm{L}}+1 / 2 \quad: \quad B(\mathrm{E} 2 ; J \rightarrow J-1)_{\mathrm{L}} \simeq\left(\frac{2 K-1}{2 K}\right)^{2} B(\mathrm{E} 2 ; J \rightarrow J-1)_{\mathrm{K}} .
\end{aligned}
$$

As yet, we have only discussed the SU(3) symmetry in the boson-fermion framework. To induce the appearance of other symmetry chains it is necessary to further restrict 
the fermion space. To take a specific and particularly fruitful example, if we choose $j=1 / 2,3 / 2,5 / 2$, then the reduction of (5.4) becomes

$$
\mathrm{U}^{\mathrm{F}}(12) \supset \mathrm{U}^{\mathrm{F}}(6) \otimes \mathrm{SU}^{\mathrm{F}}(2)
$$

The group $\mathrm{U}(6)$ now appears to describe the fermion pseudo-orbital part as well as the bosons. Algebraically, the subgroup structure of $\mathrm{U}^{\mathrm{F}}(6)$ is identical to that of $\mathrm{U}^{\mathrm{B}}(6)$ with the result that all three limiting symmetries can be realised. In fact, we are now treating our fermion space as if it arises from the coupling of $\tilde{l}=0,2$ (or $\tilde{s}, \tilde{d}$ ) states to an inert pseudo spin of $1 / 2$. Thus our boson-fermion coupling problem has become, algebraically, almost identical to the neutron-proton problem of the previous section. Exactly the same coupling options exist as in the algebra of IBM-2 but, again, experiment mandates the strongest degree of coupling at the level of $U(6)$ so that the sum group $\mathrm{U}^{\mathrm{BF}}(6)$ is obtained. The three symmetry chains of (3.1) then result, involving the bosonfermion sum groups at each stage. The structure of the solutions thus follows those of IBM-1, albeit with additional quantum numbers labelling several of the subgroups, as was found in IBM-2. For instance, states are now characterised by $\mathrm{U}^{\mathrm{BF}}(6)$ labels and, for the coupling of a single fermion to a boson core, only two possibilities arise, $[N+1,0]$ and $[N, 1]$. ¿From the parallel with IBM-2 we can recognise that these labels represent the symmetric and non-symmetric coupling of the boson and fermion orbital motion. Nevertheless, there is now a crucial physical difference between the IBFM case and that of IBM-2. In the latter, the Casimir operator of $U_{\nu \pi}(6)$ generates the symmetry energy which places the non-symmetric neutron-proton modes at a higher excitation energy. In the odd-mass case, there are no grounds for an equivalent assumption. Hence the nonsymmetric configurations can frequently constitute the ground and lowest states of the system.

An example of the $O(6)$ chain with coupling at the $U^{B F}(6)$ level is shown in figure 5.4 and illustrates many of the points made in the preceeding discussion. Firstly, the relevance of the pseudo- $L$ quantum number is evident from the empirical occurrence of couplets of levels built from each $\tilde{L}$ value. Moreover, the characteristic level sequence of an $\mathrm{O}(6)$ even-even nucleus can be discerned in the sequence of $\tilde{L}$ values, $\tilde{L}=0,2,2,4$. The two representations of $\mathrm{U}^{\mathrm{BF}}(6)$ are also evident in figure 5.4 which shows excellent agreement between theory and experiment. Similar examples-though showing lesser agreement with the data-exist for the $\mathrm{U}(5)$ and $\mathrm{SU}(3)$ symmetries. A Hamiltonian involving Casimir operators from all of the symmetry chains can also be used. A recent study of ${ }^{189}$ Os (Bruce et al., 1992) illustrates this type of approach and also highlights 


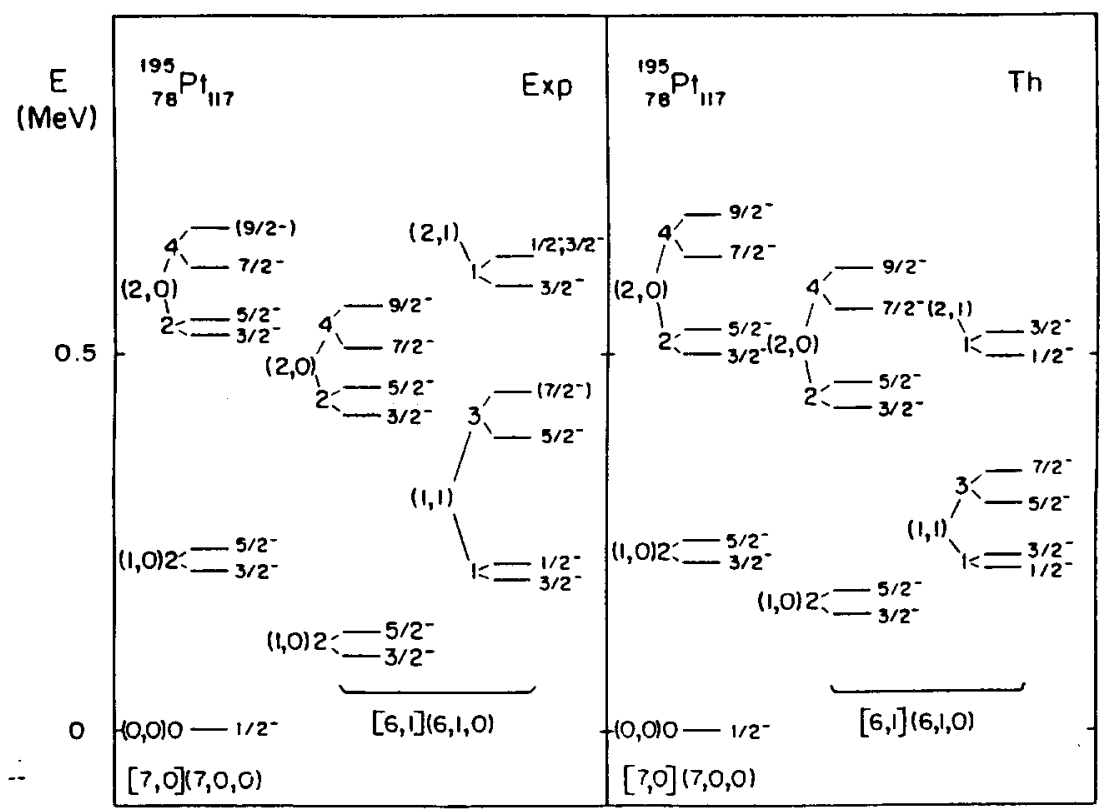

Figure 5.4: An example of a spectrum with $\mathrm{O}(6)$-type boson-fermion symmetry for orbits with $j=1 / 2,3 / 2,5 / 2:{ }^{195} \mathrm{Pt}$ (Mauthofer et al., 1986).

some of its shortcomings.

\section{Inclusion of isospin}

In section 4 it was shown how the states of a neutron-proton boson system conveniently can be classified by introducing the $F$-spin label. A single boson is assigned $F=1 / 2$ with projection $M_{\mathrm{F}}=-1 / 2$ for a neutron boson and $M_{\mathrm{F}}=+1 / 2$ for a proton boson. Although the resulting formalism is similar to that of isospin for nucleons, it was stressed that $F$ spin and isospin are not identical. This follows from the fermion-pair interpretation of the bosons: the neutron boson is made up of two neutrons and consequently has isospin $T=1$ and projection $M_{\mathrm{T}}=-1$, and similarly the proton boson has $T=M_{\mathrm{T}}=1$. This argument shows not only that $F$ spin and isospin are different labels but also that neutron and proton bosons, the building blocks of the IBM-2, do not form a complete isospin multiplet and hence that the IBM-2 is not an isospin-invariant model. (We assume throughout this section that neutrons and protons occupy the same valence shell, an assumption realised in light nuclei only. Below in this section we briefly comment on the position of isospin invariance in the IBM-2 description of heavier nuclei.) This deficiency has been remedied by the introduction of a third kind of boson, the $\delta$ boson, with $T=1$ and $M_{\mathrm{T}}=0$ and the resulting model has been named IBM-3 (Elliott 
and White, 1980). Just as $\nu$ and $\pi$ bosons, the $\delta$ boson is assumed to occupy two angular momentum states, $L=0$ and $L=2$, although effects of higher angular momentum bosons might be investigated. All bosons included in the IBM-3 have $T=1$ and, in principle, a fourth kind of boson can be introduced that corresponds to a $T=0$ neutron-proton pair. This further extension indeed has been considered (Elliott and Evans, 1981, Halse et al., 1984) and has the primary merit that it incorporates Wigner's supermultiplet theory. We will not consider it in this review.

Given that the model space of the $s d$ IBM-3 is built from three kinds of bosons each with six components, the $N$-boson states span a vector space in which the symmetric representation $[N]$ of $\mathrm{U}^{\mathrm{B}}(18)$ is defined. The classifications, containing the angular momentum as well as the isospin labels, read

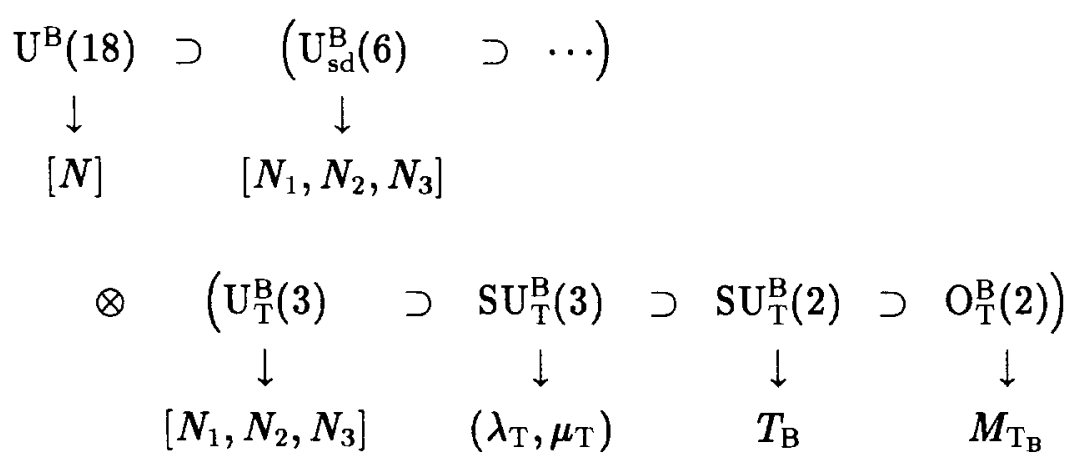

where the dots refer to one of the reductions given in (3.3). Overall symmetry of the $N$-boson wavefunction requires the representations of $\mathrm{U}_{\mathrm{sd}}^{\mathrm{B}}(6)$ and $\mathrm{U}_{\mathrm{T}}^{\mathrm{B}}(3)$ to be identical (generalised Pauli principle). Consequently, the allowed $\mathrm{U}_{\mathrm{sd}}^{\mathrm{B}}(6)$ representations can have three rows, in contrast to the IBM-1 where the representations necessarily are symmetric, and the IBM-2 where only up to two-rowed representations are allowed. The $\mathrm{SU}_{\mathrm{T}}^{\mathrm{B}}(3)$ representations are denoted in Elliott's notation, $\lambda_{\mathrm{T}}=N_{1}-N_{2}, \mu_{\mathrm{T}}=N_{2}-N_{3}$, and determine the allowed values of the isospin $T_{\mathrm{B}}$ of the bosons. Thus the choice of a particular spatial boson symmetry $\left[N_{1}, N_{2}, N_{3}\right]$ uniquely determines the allowed isospin values.

In a similar way states of a system of $M$ fermions (neutrons and protons) occupying a single- $j$ shell can be classified within the antisymmetric representation $\left[1^{M}\right]$ of $U^{\mathrm{F}}(2(2 j+$ 1)). Since, for applications in the IBFM, one is mainly interested in one-fermion states, 


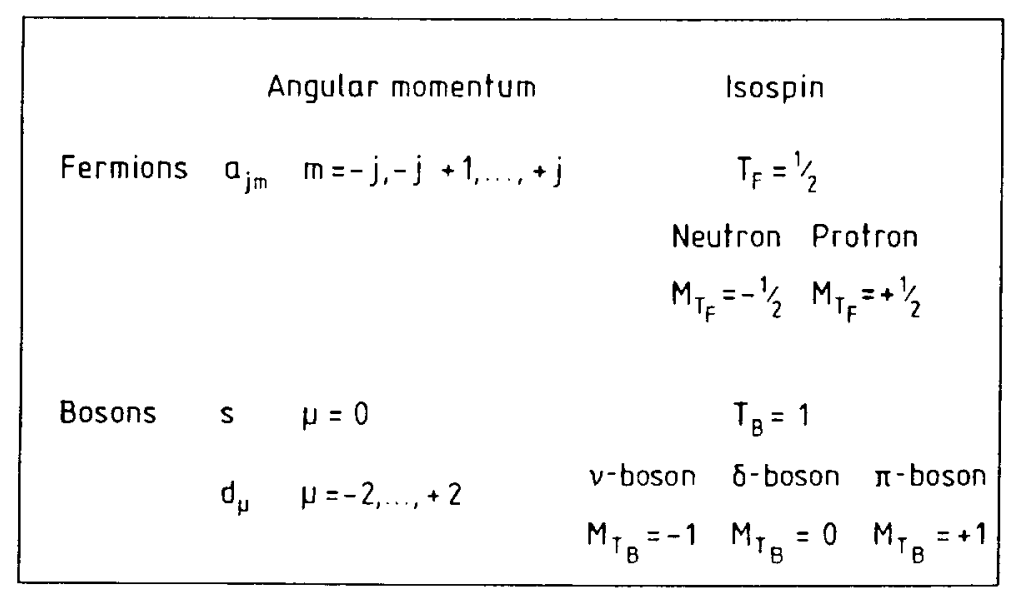

Figure 6.1: Building blocks of the IB(F)M-3 and their characterisation in angular momentum and isospin.

this classification becomes

$$
\begin{aligned}
& \mathrm{U}^{\mathrm{F}}(2(2 j+1)) \supset\left(\mathrm{U}_{\mathrm{J}}^{\mathrm{F}}(2 j+1) \supset \cdots\right) \\
& \text { [1] [1] }
\end{aligned}
$$

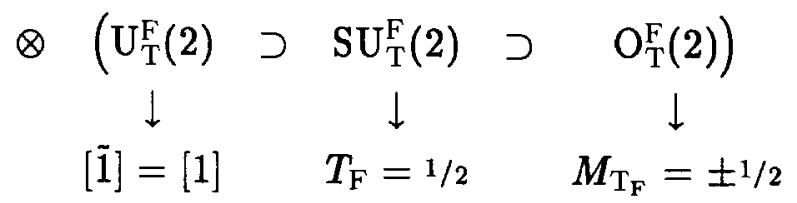

where the dots again refer to some appropriate labelling scheme for the spatial part of the wavefunction as, for instance, a seniority classification. The overall antisymmetry of the fermions requires the $\mathrm{U}_{\mathrm{T}}^{\mathrm{F}}(2)$ representation to be the conjugate of the $\mathrm{U}_{\mathrm{J}}^{\mathrm{F}}(2 j+1)$ representation (i.e. rows and columns of the Young tableau interchanged) which for one fermion is identical to itself and corresponds to isospin $T_{\mathrm{F}}=1 / 2$. Coupling of (6.1) and (6.2) in both angular momentum and isospin leads to an isospin-invariant Interacting Boson-Fermion Model (IBFM-3) (Elliott et al., 1988).

The building blocks of the IBM-3 and IBFM-3 are summarised in figure 6.1. The essential property is that complete multiplets are present both in angular momentum and isospin space, permitting the construction of operators invariant in the associated quantum numbers.

The $\delta$ boson plays an essential role in the construction of states with good isospin. To illustrate this consider the example of a state consisting of two $s$ bosons coupled to isospin $T=0$, which is a boson representation of a two-neutron-two-proton state with 
seniority $v=0$. If the $M_{\mathrm{T}}$ components of the bosons are written explicitly, i.e.

$$
\begin{aligned}
\left(s^{\dagger} s^{\dagger}\right)^{(L=0, T=0)}|\mathbf{o}\rangle & =\sum_{M_{\mathrm{T}}}\left(1 M_{\mathrm{T}} 1-M_{\mathrm{T}} \mid 00\right)\left(s_{M_{\mathrm{T}}}^{\dagger} s_{-M_{\mathrm{T}}}^{\dagger}\right)^{(L=0)}|\mathbf{o}\rangle \\
& =\left(\sqrt{\frac{2}{3}}\left(s_{\nu}^{\dagger} s_{\pi}^{\dagger}\right)^{(L=0)}-\sqrt{\frac{1}{3}}\left(s_{\delta}^{\dagger} s_{\delta}^{\dagger}\right)^{(L=0)}\right)|0\rangle
\end{aligned}
$$

one finds that a sizeable fraction $(33 \%)$ involves $\delta$ bosons. This remains true for manyparticle states but with the size of the $\delta$ component depending on the number of neutrons and protons (Thompson et al., 1987). Nuclei with $N \simeq Z$ have comparable numbers of neutrons and protons in the valence shell and will have important $\delta$ admixtures at low energies. These decrease in importance as the neutron excess increases. In fact, if the neutrons are holes (i.e. fill more than half the valence shell) and the protons are particles, the $\delta$ admixtures vanish in the limit of large $j$ (Elliott and Evans, 1987). In this case IBM-2 states approximately will have good isospin. In heavier nuclei where neutrons and protons occupy different valence shells, IBM-2 states have isospin $T=(N-Z) / 2$ and this result is exact insofar that the assumption of different valence shells for neutrons and protons is valid (Elliott, 1985). The application of the IBM-3 is thus restricted to nuclei where neutrons and protons occupy the same valence shell and where they are all particles or all holes.

The restoration of the isospin quantum number in the boson space has the advantage that the boson Hamiltonian acquires a simpler form. Without isospin invariance the introduction of the $\delta$ boson would dramatically increase the number of one- and two-body matrix elements in the Hamiltonian, just as this number increases when one goes from IBM-1 to IBM-2. Forcing the Hamiltonian to be isospin invariant, however, reduces the number of independent matrix elements and, as a consequence, IBM-2 and IBM-3 have comparable complexity, that is, they have comparable numbers of parameters.

A second, more important consequence of isospin conservation in the boson model concerns its mapping onto the fermion shell model. In the mapping procedure which is most commonly used, known as OAI mapping (Otsuka et al., 1978), the central idea is the identification of various quantum numbers in the two models and establishing a connection between them. For instance, since the model Hamiltonian in both the fermion and the boson space is rotational invariant, the resulting eigenfunctions have good angular momentum and this label can be used to associate states from the two models with one another. A second example is the seniority quantum number in fermion space (the number of fermions that are not in pairs coupled to $L=0$ ) which becomes the number of $d$ bosons in boson space. Unlike angular momentum, seniority is at best only 
an approximate quantum number, since the shell-model Hamiltonian is known to contain seniority-mixing terms that become important for nuclei with several neutrons and several protons in the valence shells. This feature of the residual nuclear interaction lies at the heart of the problems encountered in establishing the mapping between the shell model and the IBM for deformed nuclei where seniority is strongly broken. Nevertheless, for low-energy states of non-deformed nuclei seniority is an approximate quantum number in the shell model and can be used to connect its states with corresponding ones in IBM. The great advantage of the IBM-3 (as compared to IBM-1 or IBM-2) is that, besides angular momentum and seniority, also isospin can be used in establishing a mapping. For small numbers of particles these three quantum numbers will uniquely characterise all states of interest and hence define a one-to-one correspondence between shell model and IBM. Given an input shell-model Hamiltonian (deduced from experimental level energies or else some appropriate phenomenological form), its matrix elements between states with good angular momentum, seniority and isospin can be calculated and equated to corresponding matrix elements in the boson space. In this way a boson Hamiltonian is deduced from a shell-model interaction.

This procedure has been used for $f_{7 / 2}$ nuclei $(20 \leq N, Z \leq 28)$ mainly to investigate to what extent the IBM represents a valid approximation to the shell model (Thompson et al., 1987, Evans et al., 1989). In these calculations a pure $f_{7 / 2}$ shell-model space is assumed, in which case the residual interaction can be extracted from the experimental data (binding energies and/or excitation energies of $A=40,41,42$ nuclei) and exact shell-model calculations are feasible for all $20 \leq N, Z \leq 28$ nuclei. Note that a realistic description of these nuclei can only be achieved after including the effect of other orbits (such as $p_{3 / 2}$ ). For the purpose of comparing with IBM-3 and IBFM-3, however, it is better to confine the valence nucleons to the $f_{7 / 2}$ orbit. This comparison is carried out as follows. Using the mapping method explained above, shell-model calculations for $A=$ 43, 44 nuclei completely determine the boson energies and boson-boson and boson-fermion interactions. These can then be used to predict the energy spectra of $A=45-48$ nuclei and to compare IBM-3 and IBFM-3 results with 'exact' shell-model calculations for the corresponding nuclei. Two typical examples are shown in figures 6.2 and 6.3 for an eveneven (Thompson et al., 1987) and an odd-mass (Evans et al., 1989) nucleus, respectively. Note that absolute energies are calculated and that the comparison thus represents a test of the calculation of binding energies in the $\mathrm{IB}(\mathrm{F}) \mathrm{M}-3$. The connection between the shell-model and the IB(F)M-3 states, shown in figures 6.2 and 6.3 , is made on the basis 


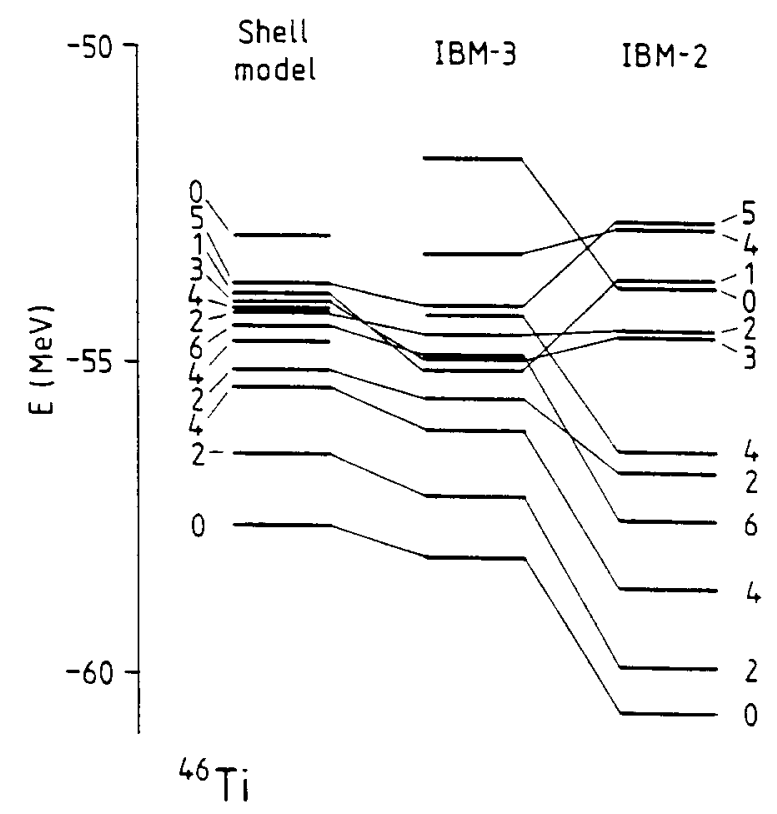

Figure 6.2: Comparison of IBM-3 and shell-model spectra for ${ }^{46} \mathrm{Ti}$ (Thompson et al., 1987). Levels are labelled by $L$.

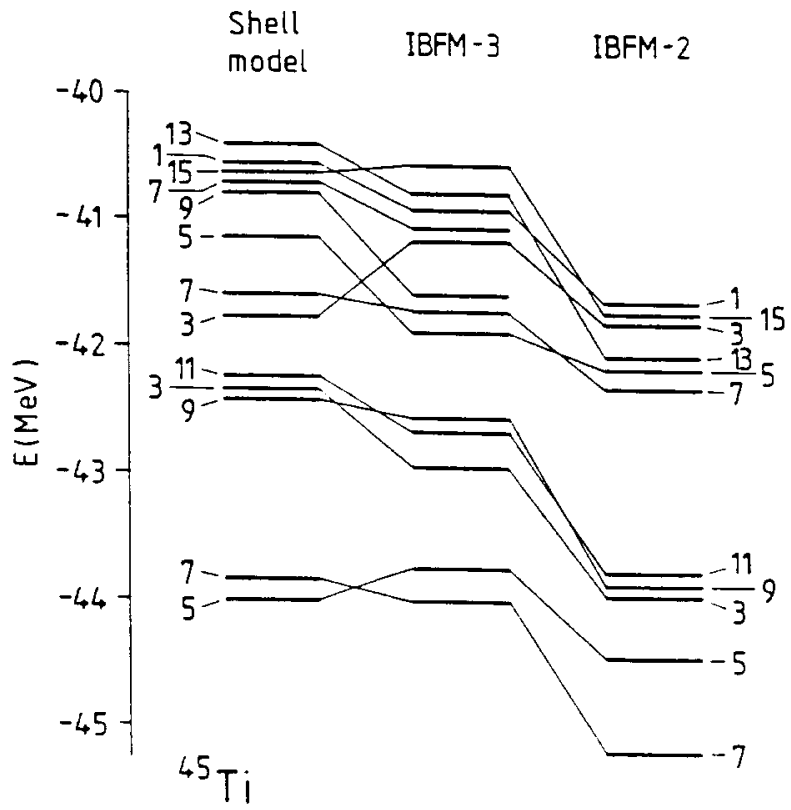

Figure 6.3: Comparison of IBFM-3 and shell-model spectra for ${ }^{45} \mathrm{Ti}$ (Evans et al., 1989). Levels are labelled by $2 J$. 
of a wavefunction analysis. The shell-model states are expanded in components with good seniority and so are IB(F)M-3 states in components with good $d$-boson number. States in the two models can be connected if corresponding components have amplitudes of similar magnitude. In general one finds that the structure of low-energy shell-model states is approximately reproduced in the IB(F)M-3. Also shown in figures 6.2 and 6.3 are the energies resulting from an $\mathrm{IB}(\mathrm{F}) \mathrm{M}-2$ calculation, where the effect of the $\delta$ boson is omitted. As argued earlier, IB(F)M-2 eigenstates do not have good isospin and it can also be seen from the figures that absolute energies are not well reproduced.

As the number of particles in the shell increases, it will not be possible, in general, to characterise a state uniquely by its angular momentum, isospin and seniority and hence no one-to-one correspondence between the shell model and the IB(F)M-3 can be established on the basis of these labels alone. To deal with such cases more quantum numbers must be defined in the shell-model space that can be carried over onto the boson space. One of them is reduced isopin $t$, the isospin of particles that are not in pairs coupled to $L=0$, which, in the IBM-3 for even-even nuclei, corresponds to the isospin $t_{\mathrm{d}}$ of the $d$ bosons. Another one is $\tilde{t}$, the isospin of the paired particles, which rather naturally maps onto the isospin $t_{\mathrm{s}}$ of the $s$ bosons. In this manner a complete mapping can be defined for all states in the $f_{7 / 2}$ shell (Evans et al., 1985). Of particular interest is the label associated with $\mathrm{U}(6)\left(\left[N_{1}, N_{2}, N_{3}\right]\right.$ in (6.1)), since this is the group that plays a basic role in the model. It can be shown (Evans et al., 1985) that IBM-3 states with well-defined U(6) quantum numbers can be written as particular combinations of states with definite $t_{\mathrm{s}}$ and $t_{\mathrm{d}}$. Using the associations $\tilde{t} \leftrightarrow t_{\mathrm{s}}$ and $t \leftrightarrow t_{\mathrm{d}}$, one may then define a $\mathrm{U}(6)$ labelling of states in the shell model and investigate whether the eigenstates of the realistic shellmodel Hamiltonian can be classified accordingly. Analysing the structure of low-energy eigenstates in even-even $f_{7 / 2}$ nuclei, it is found that in all cases $\mathrm{U}(6)$ is an approximate quantum number for such states.

This brings us to another application of the IB(F)M-3, namely the comparison of the boson(-fermion) interactions, as they are derived from the shell model, with those obtained from phenomenological fits to energy spectra of nuclei. This idea can be illustrated with the example of the Majorana interaction. In phenomenological IBM-2 calculations for even-even nuclei it is found that a boson-boson interaction is needed pushing up states with a mixed $U(6)$ symmetry (i.e. states characterised by a two-rowed $U(6)$ representation). In its simplest form this interaction has a two-body character and gives zero acting on a symmetric two-boson state [2] while it increases the energy of the antisymmetric 


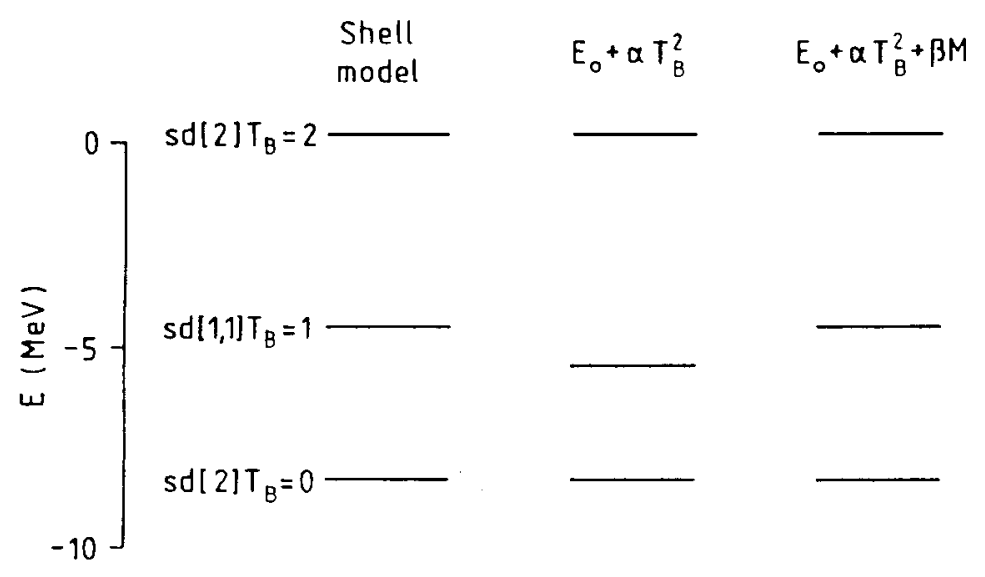

Figure 6.4: Analysis of the boson matrix elements between $s d$ states, derived from a $f_{7 / 2}$ shell-model Hamiltonian, in terms of a $T_{\mathrm{B}}^{2}$ and Majorana interaction.

state $[1,1]$ (for two bosons mixed symmetry reduces to antisymmetry); written in second quantization it is of the form (4.9). This so-called Majorana interaction $M$ is put into the model entirely on phenomenological grounds (because no empirical evidence exists for mixed-symmetry states at low energies) and it would be of interest to study it from a different perspective. Specifically, since all IBM-3 boson-boson interactions can be derived from a shell-model Hamiltonian, one may test the presence and the strength of the Majorana interaction in $f_{7 / 2}$ nuclei.

An example of such an analysis is shown in figure 6.4 where the energies of diagonal matrix elements $\left\langle s d\left[N_{1}, N_{2}\right] T_{\mathrm{B}}|V| s d\left[N_{1}, N_{2}\right] T_{\mathrm{B}}\right\rangle$, derived from the $f_{7 / 2}$ shell-model Hamiltonian, are plotted. Clearly, the matrix elements are strongly dependent on $T_{\mathrm{B}}$ making $T_{\mathrm{B}}^{2}$ the dominant contribution to the two-boson interaction. The first-order, $\mathrm{U}(6)$-symmetry breaking Hamiltonian thus acquires the form $H_{1}=E_{0}+\alpha T_{\mathrm{B}}^{2}$. The middle column of figure 6.4 shows that $H_{1}$ underestimates the energy of the $[1,1]$ states and calls for a second-order Hamiltonian of the form $H_{2}=E_{0}+\alpha T_{\mathrm{B}}^{2}+\beta M_{\mathrm{sd}}$ (column on the rhs) with positive $\beta$, where $M_{\text {sd }}$ is the first term in (4.9). This schematic analysis thus confirms the need for the repulsive Majorana force that appears in empirical fits. No quantitative conclusions can be drawn about the strength of this force in comparison with existing IBM-2 calculations, because of the difference in mass of the nuclei. Also, a more complete analysis should be carried out which includes all two-boson states and not just $s d$.

We mention that in a similar spirit the nature of the boson-fermion interaction has been studied (Evans et al., 1989). Again, it is found that the interaction, as derived from the shell model, agrees with the form used in phenomenological fits. 
We conclude this section by summarising the two principal aims of calculations carried out within the context of the isospin-invariant Interacting Boson(-Fermion) Model: (i) provide a test of the IB(F)M through comparison with exact shell-model calculations with realistic interactions and (ii) derive the IB(F)M Hamiltonian from shell-model interactions and check whether its form is consistent with Hamiltonians obtained from phenomenological fits. Both aspects have been illustrated in this section. To these we may add a third application of IB(F)M-3 which still remains to be explored, namely the prediction of properties of heavier nuclei that are difficult or impossible to describe with the shell model. Of particular interest in this respect is the $28 \leq N, Z \leq 50$ mass region where full shell-model calculations cannot be carried out in general. For a successful completion of such a programme it is of crucial importance to understand how interaction matrix elements in the IBM-3 Hamiltonian vary with neutron and proton number or, equivalently, with $N$ and $T$. For a single- $j$ shell of neutrons and protons this problem has been considered recently (Elliott et al., 1992) and, as a result of this work, the $N$ and $T$ dependence of all parameters in the IBM-3 Hamiltonian is now known, just as it is known from seniority-reduction formulas in the case of IBM-1. Based on this result, it seems that one can now attempt a systematic study of nuclei in the $28 \leq N, Z \leq 50$ region in the framework of the isospin-invariant Interacting Boson(-Fermion) Model.

\section{Scattering}

The topic of this section differs from the ones discussed in sections 3 to 6 in that it not necessarily is related to collective nuclear structure properties but rather attempts to establish a general framework for describing scattering phenomena. Nevertheless, since the methods used to arrive at this description have, in the end, a purely algebraic character and since they are akin to the approach put forward in section 2 , we believe that a discussion of them should be included in this review.

The main objective of the work summarised in this section is to formulate a scattering theory that is cast in a purely algebraic framework and, specifically, to derive the form of the $S$ matrix, as it arises in a variety of scattering problems, from Lie algebraic properties. The groups involved in this analysis are non-compact and because of this feature they are more difficult to handle mathematically than the groups we have considered so far in this review. Therefore, we choose to illustrate the approach followed in algebraic scattering theory with some simple, schematic examples. We consider the one-dimensional scattering off a Pöschl-Teller potential, related to the Lie groups $\mathrm{SU}(2)$ and $\mathrm{SU}(1,1)$, and 
by means of this example we introduce the various concepts that have a played a central role in the development of an algebraic scattering theory. We emphasise, however, that this approach is not confined to this single example: other potentials (e.g. the Morse potential) were treated in the same framework and the extension to higher dimensions (Wu et al., 1987) was considered. Since we are mainly concerned with the general approach of algebraic scattering theory, which can all be illustrated with the example of a PöschlTeller potential, we do not discuss these other topics here.

We begin our discussion by considering the Lie group $\mathrm{SU}(2)$ and its familiar realisation in terms of the angular momentum operators

$$
J_{x}=-i\left(y \frac{\partial}{\partial z}-z \frac{\partial}{\partial y}\right) \quad J_{y}=-i\left(z \frac{\partial}{\partial x}-x \frac{\partial}{\partial z}\right) \quad J_{z}=-i\left(x \frac{\partial}{\partial y}-y \frac{\partial}{\partial x}\right)
$$

which satisfy the commutation relations

$$
\left[J_{x}, J_{y}\right]=i J_{z} \quad\left[J_{y}, J_{z}\right]=i J_{x} \quad\left[J_{z}, J_{x}\right]=i J_{y}
$$

It is well known that states diagonal in $J^{2}=J_{x}^{2}+J_{y}^{2}+J_{z}^{2}$ and $J_{z}$ (i.e. eigenstates of $\left.H=\alpha J^{2}+\beta J_{z}\right)$ are characterised by the labels $j$ and $m$, with eigenvalues $j(j+1)$ and $m$, respectively. In the notation of section 2 we say that they are classified according to

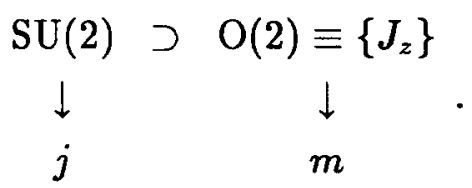

Instead of the Cartesian form (7.1) we may prefer to realise the angular momentum operators in terms of spherical coordinates

$$
x=r \sin \theta \cos \phi \quad y=r \sin \theta \sin \phi \quad z=r \cos \theta
$$

In problems with spherical symmetry the radial dependence of the wavefunctions can always be separated from the angular part $\Psi_{j m}(\theta, \phi)$ which satisfies the equations

$$
\begin{aligned}
\left(-\frac{1}{\sin \theta} \frac{\partial}{\partial \theta} \sin \theta \frac{\partial}{\partial \theta}-\frac{1}{\sin ^{2} \theta} \frac{\partial^{2}}{\partial \phi^{2}}\right) \Psi_{j m}(\theta, \phi) & =j(j+1) \Psi_{j m}(\theta, \phi) \\
-i \frac{\partial}{\partial \phi} \Psi_{j m}(\theta, \phi) & =m \Psi_{j m}(\theta, \phi) .
\end{aligned}
$$

The $\phi$-dependence is readily determined as $e^{i m \phi}, \Psi_{j m}(\theta, \phi)=\psi_{j m}(\theta) e^{i m \phi}$, with $\psi_{j m}(\theta)$ a solution of Legendre's differential equation

$$
\left(-\frac{1}{\sin \theta} \frac{d}{d \theta} \sin \theta \frac{d}{d \theta}+\frac{m^{2}}{\sin ^{2} \theta}\right) \psi_{j m}(\theta)=j(j+1) \psi_{j m}(\theta)
$$




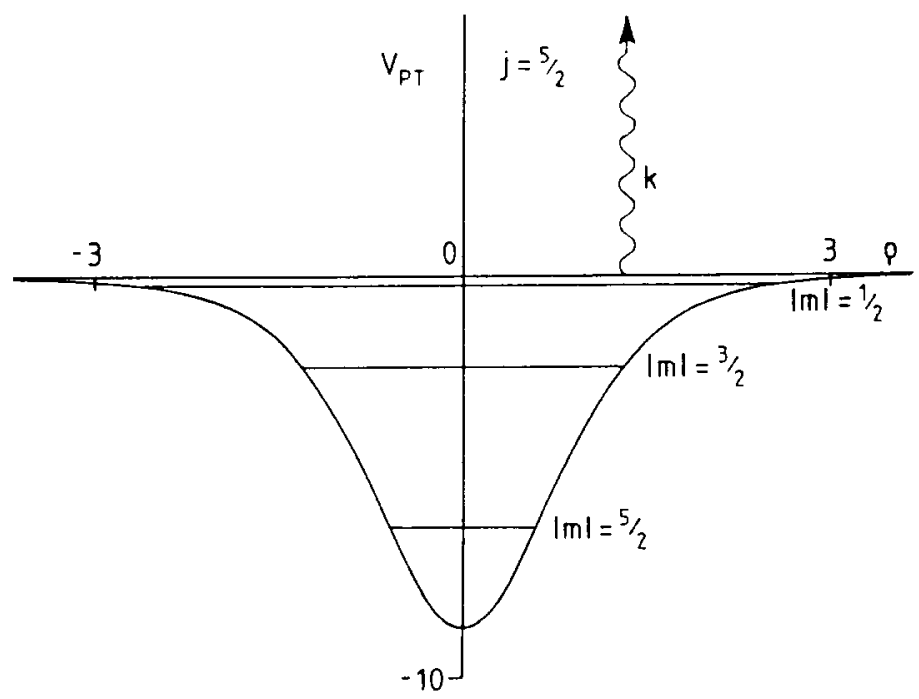

Figure 7.1: Pöschl-Teller potential with strength $V_{0}=\frac{35}{4}$. Bound states are shown as horizontal straight lines; the continuum is indicated with a wavy line.

Introducing the variable $\rho$ through the relation $\cos \theta=\tanh \rho$, with $\rho$ defined over the domain $-\infty<\rho<+\infty$, we arrive at

$$
\left(-\frac{d^{2}}{d \rho^{2}}-\frac{j(j+1)}{\cosh ^{2} \rho}\right) \varphi_{j m}(\rho)=-m^{2} \varphi_{j m}(\rho)
$$

with $\varphi_{j m}(\rho)=\psi_{j m}(\theta)$. This is the one-dimensional Schrödinger equation for the bound states of the Pöschl-Teller potential $V_{\mathrm{PT}}(\rho)=-V_{0} / \cosh ^{2} \rho$ (see figure 7.1) with strength $V_{0}=j(j+1)$. The derivation leading to (7.7) shows that the properties of this potential are intimately connected with the algebraic structure of $\mathrm{SU}(2)$. We also note from (7.7) that SU(2) plays the role of a dynamical group for the bound states of the Pöschl-Teller potential: the representation $j$ of $\mathrm{SU}(2)$, which defines the strength of the potential, contains all bound states $|m|=j,(j-1), \ldots, \frac{1}{2}$ or 0 for that particular strength.

Since $m$ is real, equation (7.7) contains the bound states of the Pöschl-Teller potential and should thus be modified to include scattering states. This can be achieved via the analytic continuation $m \rightarrow i k$. The first step towards an algebraic formulation of scattering theory is due to Alhassid et al. (1983) who showed that this analytic continuation is equivalent to the introduction of the non-compact group $\mathrm{SU}(1,1)$ to replace $\mathrm{SU}(2)$. The way to get from $\mathrm{SU}(1,1)$ to the Pöschl-Teller scattering states is very similar to the derivation (7.1)-(7.7), but at the same time some important differences occur between 
the two cases. An $\mathrm{SU}(1,1)$ group can be realised in terms of the operators

$$
I_{x}=i\left(y \frac{\partial}{\partial z}+z \frac{\partial}{\partial y}\right) \quad I_{y}=-i\left(z \frac{\partial}{\partial x}-x \frac{\partial}{\partial z}\right) \quad I_{z}=-i\left(x \frac{\partial}{\partial y}+y \frac{\partial}{\partial x}\right)
$$

which satisfy the commutation relations

$$
\left[I_{x}, I_{y}\right]=i I_{z} \quad\left[I_{y}, I_{z}\right]=i I_{x} \quad\left[I_{z}, I_{x}\right]=-i I_{y}
$$

The non-compactness of the group arises because of the minus sign in the last of the commutation relations. The Casimir operator of $\mathrm{SU}(1,1)$ is $I^{2}=-I_{x}^{2}+I_{y}^{2}-I_{z}^{2}$ (with eigenvalue $j(j+1)$ ) and, as in the $\mathrm{SU}(2)$ case, we want to find the states that simultaneously diagonalise $I^{2}$ and $I_{z}$. This can be achieved most conveniently by introducing the hyperbolic coordinates

$$
x=r \sin \theta \cosh \phi \quad y=r \sin \theta \sinh \phi \quad z=r \cos \theta
$$

Note that, in contrast to the $\mathrm{SU}(2)$ case, the non-compactness of $\mathrm{SU}(1,1)$ leads to a variable $\phi$ with an unbounded domain $(-\infty<\phi<+\infty)$. Writing $I^{2}$ and $I_{z}$ in terms of the hyperbolic coordinates, we find that they are independent of $r$ and lead to the 'angular' eigenequations

$$
\begin{aligned}
\left(-\frac{1}{\sin \theta} \frac{\partial}{\partial \theta} \sin \theta \frac{\partial}{\partial \theta}+\frac{1}{\sin ^{2} \theta} \frac{\partial^{2}}{\partial \phi^{2}}\right) \Psi_{j k}(\theta, \phi) & =j(j+1) \Psi_{j k}(\theta, \phi) \\
-i \frac{\partial}{\partial \phi} \Psi_{j k}(\theta, \phi) & =k \Psi_{j k}(\theta, \phi)
\end{aligned}
$$

and, after the transformation $\cos \theta=\tanh \rho(-\infty<\rho<+\infty)$, we arrive at the PöschlTeller equation

$$
\left(-\frac{d^{2}}{d \rho^{2}}-\frac{j(j+1)}{\cosh ^{2} \rho}\right) \varphi_{j k}(\rho)=k^{2} \varphi_{j k}(\rho)
$$

In this case the classification analogous to (7.3) reads

$$
\begin{array}{cc}
\mathrm{SU}(1,1) & \supset \\
\downarrow & \mathrm{O}(1,1) \equiv\left\{I_{z}\right\} \\
j & \downarrow
\end{array} .
$$

The eigenstates $\Psi_{j k}(\theta, \phi)$ are diagonal in the non-compact generator $I_{z}$ (non-compact since the associated variable $\phi$ is unbounded). The allowed values of $k$ for a given $j$ are thus found from the branching rule $\mathrm{SU}(1,1) \supset \mathrm{O}(1,1)$ and it turns out that $k$ must be real but is arbitrary otherwise. That $k$ is continuous rather than discrete can be understood 
intuitively as follows. The restriction of a quantum number to discrete values always follows from a periodic boundary condition; since $\phi$ is unbounded, no such condition can be imposed and hence $k$ is continuous. For real, continuous $k$ equation (7.12) describes Pöschl-Teller scattering states and hence we have achieved our objective of connecting such states with a Lie group. Again, $\mathrm{SU}(1,1)$ plays the role of a dynamical group since, for a given potential with strength $j(j+1)$, the complete continuum is contained in a single representation $j$ of $\mathrm{SU}(1,1)$.

The situation is summarised in figure 7.1 in the case of a Pöschl-Teller potential with $j=\frac{5}{2}$ (strength $V_{0}=\frac{35}{4}$ ). Three bound states occur corresponding with $|m|=\frac{5}{2}, \frac{3}{2}$ and $\frac{1}{2}$, contained in the $j=\frac{5}{2}$ representation of $\mathrm{SU}(2)$. The continuum states, indicated with a wavy line and characterised by the real variable $k$, are contained in the $j=\frac{5}{2}$ representation of $\mathrm{SU}(1,1)$.

This procedure for linking scattering states to a non-compact dynamical group, although extendable to other potentials and to higher dimensions, has the distinct disadvantage that bound and unbound eigenstates are contained in different dynamical groups. In the example of the Pöschl-Teller potential these are $\mathrm{SU}(2)$ and $\mathrm{SU}(1,1)$. It would be desirable, for systems with mixed spectra, to have a common dynamical group. In a subsequent development of algebraic scattering theory Frank and Wolf (1984) showed that this indeed is possible. Their analysis starts from the following realisation of $\mathrm{SU}(1,1)$ :

$$
N_{x}=-i\left(y \frac{\partial}{\partial z}+z \frac{\partial}{\partial y}\right) \quad N_{y}=i\left(z \frac{\partial}{\partial x}+x \frac{\partial}{\partial z}\right) \quad N_{z}=-i\left(x \frac{\partial}{\partial y}-y \frac{\partial}{\partial x}\right)
$$

These operators satisfy the commutation relations

$$
\left[N_{x}, N_{y}\right]=-i N_{z} \quad\left[N_{y}, N_{z}\right]=i N_{x} \quad\left[N_{z}, N_{x}\right]=i N_{y}
$$

and define the Casimir operator $N^{2}=-N_{x}^{2}-N_{y}^{2}+N_{z}^{2}$. To simultaneously diagonalise $N^{2}$ and $N_{z}$ we introduce the polar hyperbolic coordinates

$$
x=r \cosh \rho \cos \phi \quad y=r \cosh \rho \sin \phi \quad z=r \sinh \rho
$$

and, after a similarity transformation in $\rho$, we arrive at the eigenequations

$$
\begin{aligned}
{\left[\frac{\partial^{2}}{\partial \rho^{2}}-\frac{1}{\cosh ^{2} \rho}\left(\frac{\partial^{2}}{\partial \phi^{2}}+\frac{1}{4}\right)\right.} & \left.-\frac{1}{4}\right] \Psi_{j m}(\rho, \phi)=j(j+1) \Psi_{j m}(\rho, \phi) \\
-i \frac{\partial}{\partial \phi} \Psi_{j m}(\rho, \phi) & =m \Psi_{j m}(\rho, \phi)
\end{aligned}
$$


With $\Psi_{j m}(\rho, \phi)=\varphi_{j m}(\rho) e^{i m \phi}$ we obtain the following differential equation in $\rho$ :

$$
\left(-\frac{d^{2}}{d \rho^{2}}-\frac{m^{2}-\frac{1}{4}}{\cosh ^{2} \rho}\right) \varphi_{j m}(\rho)=-\left(j+\frac{1}{2}\right)^{2} \varphi_{j m}(\rho) .
$$

Several important differences arise between the present realisation of $\mathrm{SU}(1,1)$ and the one in terms of $I_{x}, I_{y}$ and $I_{z}$. In the first place, we note that $\phi$ is now defined over a bounded domain $(0 \leq \phi<4 \pi)$. The associated operator, $N_{z}$, is thus a compact operator and the eigenstates $\Psi_{j m}(\rho, \phi)$ are classified according to

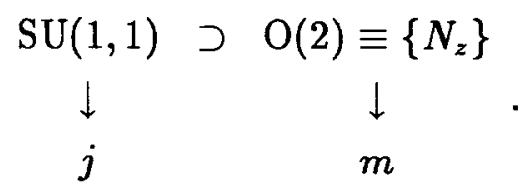

Since $m$ is associated with a compact generator, it is a discrete variable.

Secondly, two types of unitary irreducible representations $j$ of $\mathrm{SU}(1,1)$ occur (Wybourne, 1974) that have physical relevance in the Pöschl-Teller equation (7.18):

1. Discrete series $D_{j}^{\sigma}(\sigma= \pm)$ with $j$ a negative integer or half-integer. The allowed values of $m$ are

$$
m=\left\{\begin{array}{ll}
-j,-j+1,-j+2, \ldots & \sigma=+ \\
j, j-1, j-2, \ldots & \sigma=-
\end{array} .\right.
$$

Since $-m$ and $m$ correspond to the same potential (7.18), we need not distiguish between the two cases $\sigma=-$ and $\sigma=+$.

2. Continuous series $C_{k}^{\tau}\left(\tau=0, \frac{1}{2}\right)$ with $j=-\frac{1}{2}+i k, k=$ real $>0$. The allowed values of $m$ in this case are

$$
m=\left\{\begin{array}{ll}
0, \pm 1, \pm 2, \ldots & \tau=0 \\
\pm \frac{1}{2}, \pm \frac{3}{2}, \ldots & \tau=\frac{1}{2}
\end{array} .\right.
$$

The regions in the complex $j$ plane occupied by unitary irreducible representations of $\mathrm{SU}(1,1)$ having a physical interpretation are indicated in figure 7.2 , either by a cross (discrete) or by a wavy line (continuum).

Another important difference with respect to the previous realisation is the interchange in the role of $j$ and $m$. In the Pöschl-Teller equation (7.18) the strength of the potential depends on $m$ and the energy spectrum is determined by the associated values for $j$. As a consequence, $\mathrm{SU}(1,1)$ has lost its role as the dynamical group of the problem. One representation $j$ of $S U(1,1)$ now contains states with the same energy but with different potential strengths, and hence $\mathrm{SU}(1,1)$ can be characterised as the potential group (Frank and Wolf, 1984). 


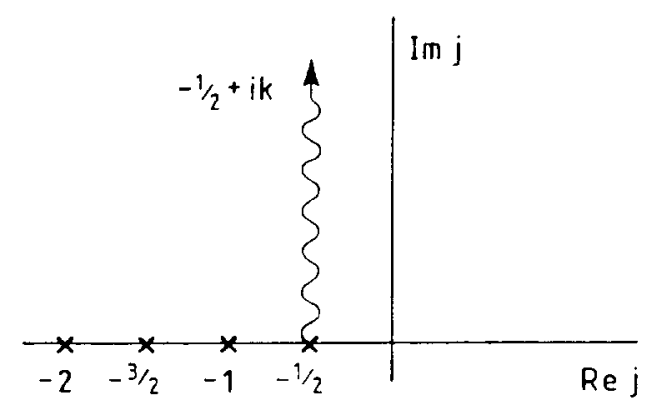

Figure 7.2: The regions of the complex $j$ plane occupied by unitary irreducible representations of $\mathrm{SU}(1,1)$. A cross indicates a discrete state and the wavy line represents the continuum.

The complete eigenspectrum of a Pöschl-Teller potential (bound and continuum states) now follows from the classification of the discrete and continuous series. For a fixed $m$ (positive integer or half-integer) the bound-state spectrum is contained in the discrete series $D_{j}^{+}$,

$$
E_{j}=-\left(j+\frac{1}{2}\right)^{2} \quad j=\left\{\begin{array}{lll}
-\frac{1}{2},-\frac{3}{2}, \ldots,-m & m \text { half-integer } \\
-1,-2, \ldots,-m & m \text { integer }
\end{array}\right.
$$

with the discrete series $D_{j}^{-}$giving equivalent results. For a fixed, positive, integer (halfinteger) $m$ the continuum states are contained in the continuous series $C_{k}^{0}\left(C_{k}^{1 / 2}\right)$ :

$$
E_{k}=k^{2} \quad k=\text { real }>0 .
$$

The role of the potential group is illustrated in figure 7.3 where several Pöschl-Teller potentials are shown together with their energy spectra. One representation $j$ (integer or half-integer $<0)$ of $\mathrm{SU}(1,1)$ contains bound states with the same energy $-\left(j+\frac{1}{2}\right)^{2}$ belonging to Pöschl-Teller potentials with $m=-j,-j+1, \ldots$ (connected by a dashed line in figure 7.3). The corresponding situation for continuum states is also indicated.

Although a connection between the bound and continuum states of the Pöschl-Teller potential and the group $\mathrm{SU}(1,1)$ is now clearly established, it should be noted that our main goal-an algebraic derivation of the $S$ matrix - is not yet achieved. Of course, in the case of the Pöschl-Teller potential the solutions $\varphi_{j m}(\rho)$ are known in terms of standard mathematical functions from which the $S$ matrix can be obtained. The whole point of the excercise is, however, not to use this explicit coordinate representation and derive the $S$ matrix from the properties of SU(1,1). We now proceed to show how this can be done, following the approach proposed by Alhassid et al. $(1984,1986)$. Their analysis makes use of the SU(1,1) realisation (7.14) in terms of $N_{x}, N_{y}$ and $N_{z}$. After a transformation 

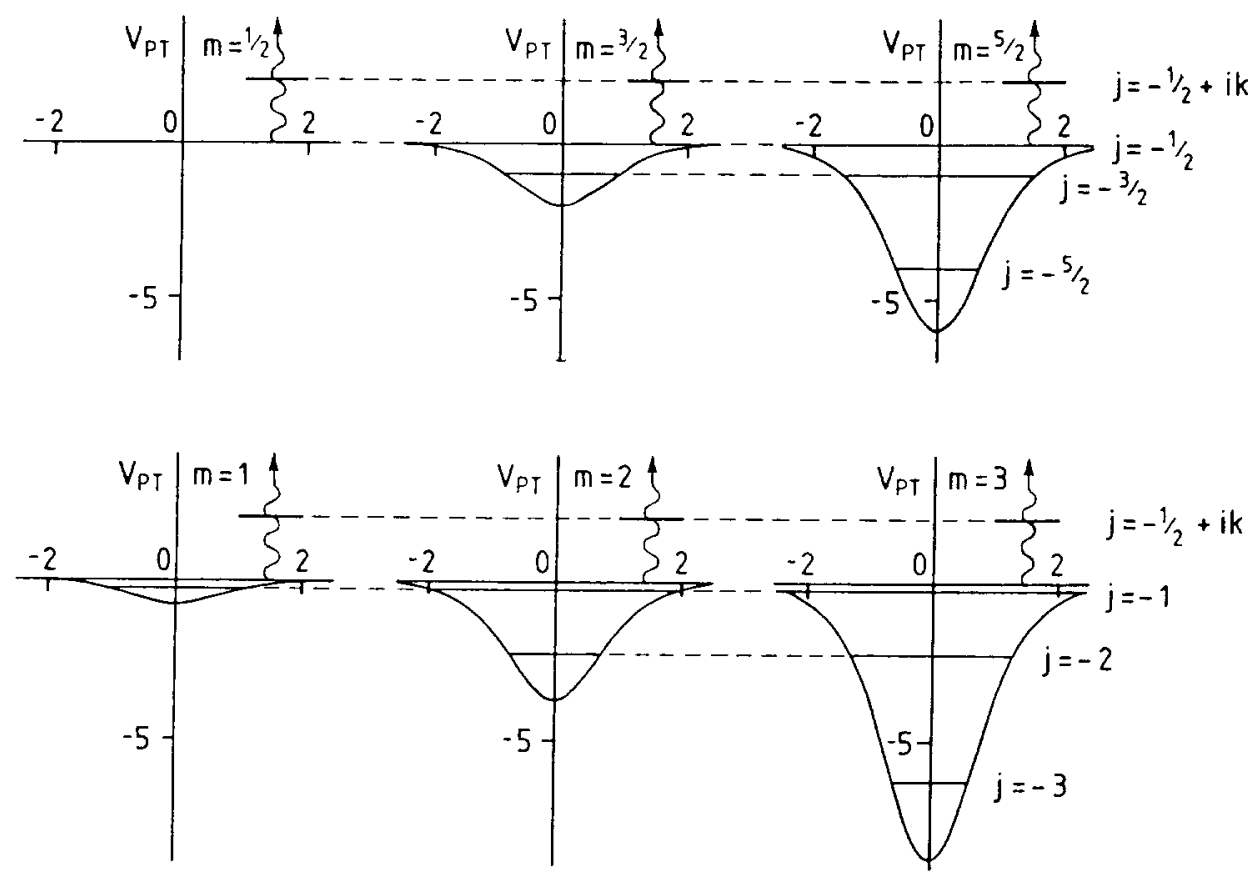

Figure 7.3: Pöschl-Teller potentials with different strengths and their connection implied by the potential group. Bound states are shown as horizontal straight lines; the continuum is indicated with a wavy line.

to the polar hyperbolic coordinates (7.16) these generators become

$$
N_{ \pm}=e^{ \pm i \phi}\left[\mp \frac{\partial}{\partial \rho}+\tanh \rho\left( \pm \frac{1}{2}-i \frac{\partial}{\partial \phi}\right)\right] \quad N_{z}=-i \frac{\partial}{\partial \phi}
$$

where the operators $N_{ \pm}=N_{x} \pm i N_{y}$ are introduced. The central element in the method of Alhassid et al. (1986) is to study the behaviour of these generators as $\rho \rightarrow \infty$, which is easily obtained from (7.24):

$$
\begin{aligned}
& N_{ \pm}^{\infty} \equiv \lim _{\rho \rightarrow \infty} N_{ \pm}=e^{ \pm i \phi}\left[\mp \frac{\partial}{\partial \rho}+\left( \pm \frac{1}{2}-i \frac{\partial}{\partial \phi}\right)\right] \\
& N_{z}^{\infty} \equiv \lim _{\rho \rightarrow \infty} N_{z}=-i \frac{\partial}{\partial \phi} .
\end{aligned}
$$

These operators still satisfy $\mathrm{SU}(1,1)$ commutation relations and we denote the associated group as $\mathrm{SU}^{\infty}(1,1)$. Likewise, the asymptotic behaviour of the wavefunctions $\Psi_{j m}(\rho, \phi)$ can be studied:

$$
\begin{aligned}
\Psi_{j m}^{\infty}(\rho, \phi) & \equiv \lim _{\rho \rightarrow \infty} \Psi_{j m}(\rho, \phi)=\lim _{\rho \rightarrow \infty} \varphi_{j m}(\rho) e^{i m \phi} \\
& =A_{m} e^{-i k \rho} e^{i m \phi}+B_{m} e^{i k \rho} e^{i m \phi}
\end{aligned}
$$


The last form in (7.26) is appropriate for a wavefunction with positive energy $E_{k}=k^{2}$ (with $j=-\frac{1}{2}+i k$ ) which we furthermore assume to be coming from the right. This general form is valid for any (one-dimensional) potential $V(\rho)$ that reduces to a constant for $\rho \rightarrow \pm \infty$. The coefficients $A_{m}$ and $B_{m}$ depend on the form of the potential and determine the structure of the $S$ matrix, e.g. the ratio $B_{m} / A_{m}$ gives the reflection amplitude $R_{m}$ for a wave scattered by the potential. To reiterate the point made earlier, $R_{m}$ can be obtained using the explicit form of $\Psi_{j m}(\rho, \phi)$ but it is our aim here to derive it from the algebraic properties of $\mathrm{SU}(1,1)$.

The second ingredient in the method of Alhassid et al. (1986) is the algebraic characterisation of the incoming and outgoing wavefunctions in (7.26). With this purpose in mind the Euclidean group in two dimensions, $\mathrm{E}(2)$, is introduced which can be realised in terms of the operators

$$
P_{x}=-i \frac{\partial}{\partial x} \quad P_{y}=-i \frac{\partial}{\partial y} \quad L_{z}=-i\left(x \frac{\partial}{\partial y}-y \frac{\partial}{\partial x}\right)
$$

satisfying the commutation relations

$$
\left[P_{+}, P_{-}\right]=0 \quad\left[L_{z}, P_{ \pm}\right]= \pm P_{ \pm}
$$

where $P_{ \pm}=P_{x} \pm i P_{y}$. Again, to find the wavefunctions diagonal in $P^{2}=P_{x}^{2}+P_{y}^{2}$ and $L_{z}$ (Casimir operators of $\mathrm{E}(2)$ and $\mathrm{O}(2)$ ) a transformation to polar coordinates is carried out

$$
x=\rho \cos \phi \quad y=\rho \sin \phi
$$

and, after a similarity transformation, the following eigenequations are obtained:

$$
\begin{aligned}
{\left[-\frac{\partial^{2}}{\partial \rho^{2}}-\frac{1}{\rho^{2}}\left(\frac{\partial^{2}}{\partial \phi^{2}}+\frac{1}{4}\right)\right] \Upsilon_{k m}(\rho, \phi) } & =k^{2} \Upsilon_{k m}(\rho, \phi) \\
-i \frac{\partial}{\partial \phi} \Upsilon_{k m}(\rho, \phi) & =m \Upsilon_{k m}(\rho, \phi)
\end{aligned}
$$

with solutions $\Upsilon_{k m}(\rho, \phi)$ classified according to

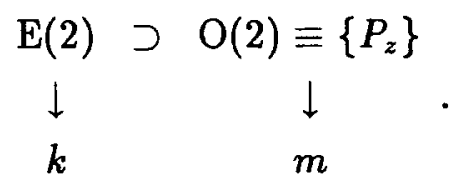

The explicit expressions for the wavefunctions $\Upsilon_{k m}(\rho, \phi)$ are of no importance here but rather their asymptotic behaviour as $\rho \rightarrow \infty$. From

$$
P_{ \pm}= \pm e^{ \pm i \phi}\left[ \pm \frac{\partial}{\partial \rho} \pm \frac{1}{\rho}\left( \pm \frac{1}{2}-i \frac{\partial}{\partial \phi}\right)\right] \quad L_{z}=-i \frac{\partial}{\partial \phi}
$$


one finds the asymptotic forms

$$
P_{ \pm}^{\infty} \equiv \lim _{\rho \rightarrow \infty} P_{ \pm}=-e^{ \pm i \phi} \frac{\partial}{\partial \rho} \quad L_{z}^{\infty} \equiv \lim _{\rho \rightarrow \infty} L_{z}=-i \frac{\partial}{\partial \phi}
$$

which shows that the incoming and outgoing wavefunctions $e^{ \pm i k \rho} e^{i m \phi}$ are simultaneous eigenstates of $\left(P^{\infty}\right)^{2}$ and $L_{z}$. Thus the algebraic characterisation of the incoming and outgoing wavefunctions in (7.26) is that they arise as the asymptotic form of the wavefunctions classified according to $\mathrm{E}(2) \supset \mathrm{O}(2)$.

The last step in the analysis of Alhassid et al. (1986) involves establishing a connection between the asymptotic generators of $\mathrm{SU}(1,1)$ and $\mathrm{E}(2)$, and is known as the Euclidean connection. Relations (7.25) and (7.33) show that the generators of $\mathrm{SU}^{\infty}(1,1)$ can be expressed in terms of those of $\mathrm{E}^{\infty}(2)$ :

$$
N_{+}^{\infty}(k)=\frac{1}{k}\left[\left(-\frac{1}{2}-i k\right) P_{+}^{\infty}+L_{z}^{\infty} P_{+}^{\infty}\right]
$$

and, furthermore, $N_{-}^{\infty}=\left(N_{+}^{\infty}\right)^{\dagger}$ and $N_{z}^{\infty}=L_{z}^{\infty}$. These relations are valid under the assumption that the operators act on the asymptotic wavefunctions $e^{ \pm i k \rho} e^{i m \phi}$ and, because of this, $k$ appears as a parameter. The $S$ matrix of the Pöschl-Teller potential can now be derived on the basis of the connection formula. First, we use the property that $N_{+}^{\infty}$ is a raising operator of $\mathrm{SU}^{\infty}(1,1)$ :

$$
N_{+}^{\infty} \Psi_{j m}^{\infty}(\rho, \phi)=\sqrt{(m-j)(m+j+1)} \Psi_{j m+1}^{\infty}(\rho, \phi) .
$$

We then expand $\Psi_{j m}^{\infty}(\rho, \phi)$ and $\Psi_{j m+1}^{\infty}(\rho, \phi)$ according to (7.26) and evaluate the action of $N_{+}^{\infty}$ on the lhs using the connection formula (7.34). Equating corresponding terms we obtain the recurrence relations

$$
\begin{aligned}
& \sqrt{(m-j)(m+j+1)} A_{m+1}=\left(m+\frac{1}{2}+i k\right) A_{m} \\
& \sqrt{(m-j)(m+j+1)} B_{m+1}=\left(m+\frac{1}{2}-i k\right) B_{m} .
\end{aligned}
$$

Since $R_{m}=B_{m} / A_{m}$, a recurrence relation for the reflection coefficient $R_{m}$ is obtained which, given an appropriate initial condition, can be solved.

We emphasise that in this derivation of the $S$ matrix no use is made of the explicit expression for the wavefunctions but only of their asymptotic behaviour (which is generally valid), the algebraic properties of $\mathrm{SU}(1,1)$ and the connection formula (7.34). The crucial point of the method is that the connection formula can be readily generalised to other potentials with the sole assumption that the Hamiltonian is an arbitrary function $h$ of 


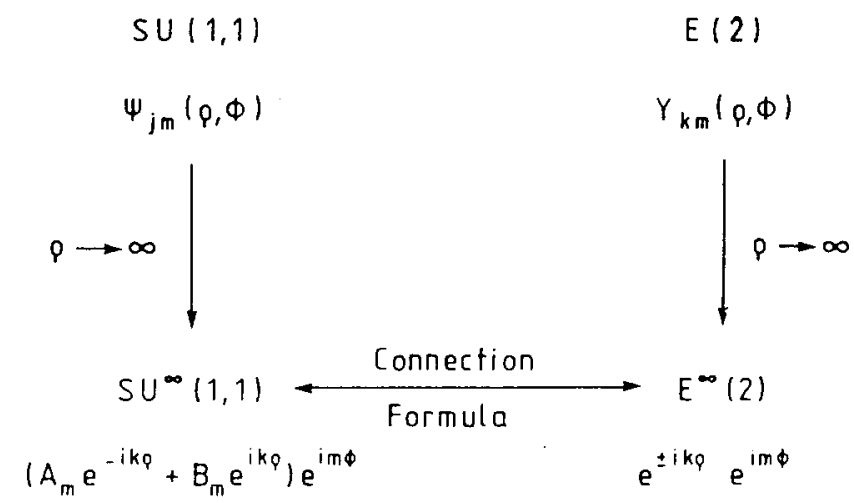

Figure 7.4: Summary of groups, associated wavefunctions and their asymptotic expressions used in the connection formula.

the Casimir operator of $\mathrm{SU}(1,1)$. Under this restriction Alhassid et al. (1986) are able to derive the more general connection formula

$$
N_{+}^{\infty}( \pm k)= \pm \frac{e^{i \gamma_{ \pm}(k)}}{k}\left[\left(-\frac{1}{2} \mp i f(k)\right) P_{+}^{\infty}+L_{z}^{\infty} P_{+}^{\infty}\right]
$$

where $\gamma_{ \pm}(k)$ are two arbitrary, real functions of $k$ and $f(k)$ is determined by the potential and related to functional form of $h$. This connection formula then leads to the recurrence relations

$$
\begin{aligned}
& \sqrt{(m-j)(m+j+1)} A_{m+1}=e^{i \gamma_{-}(k)}\left(m+\frac{1}{2}+i f(k)\right) A_{m} \\
& \sqrt{(m-j)(m+j+1)} B_{m+1}=e^{i \gamma_{+}(k)}\left(m+\frac{1}{2}-i f(k)\right) B_{m}
\end{aligned}
$$

from which one obtains the reflection amplitude

$$
R_{m}(k)=e^{i\left[\gamma_{+}(k)-\gamma_{-}(k)\right] m} \frac{\Gamma\left(m+\frac{1}{2}-i f(k)\right) \Gamma\left(m_{0}+\frac{1}{2}+i f(k)\right)}{\Gamma\left(m+\frac{1}{2}+i f(k)\right) \Gamma\left(m_{0}+\frac{1}{2}-i f(k)\right)} R_{m_{0}}(k)
$$

where $m_{0}$ is the fractional part of $m$ and $R_{m_{0}}(k)$ is an entire function (no singularities) in the complex $k$ plane. In many cases this function is known explicitly. Equation (7.39) is the most general expression for the reflection amplitude in a problem with $\mathrm{SU}(1,1)$ symmetry.

The derivation leading to the connection formula (7.37) and the reflection amplitude (7.39) is rather long and technical, and it is therefore useful to summarise here the main points of the method (see figure 7.4).

1. The potential group $\mathrm{SU}(1,1)$ and the wavefunctions $\Psi_{j m}(\rho, \phi)$ are studied in the limit $\rho \rightarrow \infty$. No explicit coordinate realisation is needed to find the general form of the asymptotic wavefunctions in terms of incoming and outgoing waves. 
2. The Euclidean group $\mathrm{E}(2)$ and its wavefunctions $\Upsilon_{k m}(\rho, \phi)$ are studied in the limit $\rho \rightarrow \infty$. It is shown that incoming and outgoing waves $e^{ \pm i k \rho} e^{i m \phi}$ are classified according $\mathrm{E}^{\infty}(2) \supset \mathrm{O}^{\infty}(2)$.

3. The connection formula is established. Under the assumption of $S U(1,1)$ symmetry the generators of $\mathrm{SU}^{\infty}(1,1)$ can be written in terms of those of $\mathrm{E}^{\infty}(2)$.

4. The concept of $\mathrm{SU}(1,1)$ as a potential group is used. Action of the raising operator $N_{+}^{\infty}$ relates the asymptotic behaviour of wavefunctions corresponding to the potentials $m$ and $m+1$ and hence recurrence relations are derived for $A_{m}$ and $B_{m}$. In this derivation the $\mathrm{SU}(1,1)$ raising property of $N_{+}^{\infty}$ is used as well as its connection with the $\mathrm{E}^{\infty}(2)$ generators, but an explicit coordinate realisation is not needed.

We conclude this discussion by noting that the procedure for establishing the Euclidean connection has been cast in a more abstract form using techniques of group contractions and expansions (Frank et al., 1986) allowing for a systematic investigation of possible connection formulae.

In contrast to the discussion in the preceeding sections, the material reviewed here is of a more formal nature and less concerned with immediate applications to realistic reaction processes. Nevertheless, the formal derivation of algebraic scattering theory was followed by initial attempts to investigate realistic heavy-ion reactions (Alhassid and Iachello, 1989) and, in particular, the problem of the coupling of several reaction channels was considered. More recently, applications have been reported (Lépine-Szily et al., 1990, 1992), which reveal some of the potential of algebraic scattering theory: because the coupled-channels problem reduces to a simple matrix diagonalisation, a large number of channels can be considered in the calculation. It this way it is possible to describe the back-angle anomaly in the ${ }^{12} \mathrm{C}+{ }^{24} \mathrm{Mg}$ system (Lépine-Szily et al., 1990) as well as orbiting phenomena in the ${ }^{18} \mathrm{O}+{ }^{10,11} \mathrm{~B}$ reaction (Lépine-Szily et al., 1992).

\section{Summary and conclusions}

This review has been focussed on the family of models which have grown out of the basic framework represented by the IBM. An introduction to the basic idea of dynamical symmetry and some familiar examples of its use in different disciplines, has been followed by a brief reminder of the simplest version of the model, which treats the collective structure in terms of a single species of boson occupying two possible angular momentum 
states. We have then dealt with its extension to the explicit recognition of neutron and proton degrees of freedom, the coupling of an odd particle, the inclusion of $M_{\mathrm{T}}=0$ bosons and finally to the application of algebraic techniques to scattering phenomena. The ordering of topics has thus followed the historical development of the model. The examples discussed have been chosen to illustrate the algebraic origin and construction of the Hamiltonian in each case.

The overall approach has, by now, led to a rather complete and extensive framework in which most facets of collective nuclear structure can be treated. Moreover, the underlying assumption upon which the IBM is based must not be overlooked. The bosonic basis can be linked to the underlying fermion one by means of mapping techniques which describe the $s$ - and $d$-boson states in terms of a correlated superposition of two-quasi-particle states. The review by Iachello and Talmi (1987) describes some of this work. This link lends the algebraic approach discussed here a microscopic aspect in many of its predictions, by virtue of the dependence on valence particle number incorporated in the boson number dependence of many quantities and by the possibility to associate the appearance and disappearance of specific symmetries with the single-particle structure. Indeed, this is also one of the aims of the FDSM, which has taken an alternative approach of defining symmetries in the fermion basis itself and then attempting to reproduce, and if possible to go beyond, the results of the IBM.

The empirical evidence is irrefutable in respect of confirming the role played by symmetry in collective nuclear structure. Nevertheless, it should be emphasised that there are relatively few examples of the symmetry structures in 'exact' form. Rather they define standard structures in the same way as the terms 'vibrational' and 'rotational' do in a geometrical context. A more exact description frequently requires a numerical calculation which effectively involves the Casimir operators of two or more symmetries.

In this final paragraph, it may be worthwhile to say something about the future. All branches of the IBM are still being developed and that is no less true of many other nuclear structure models currently in existence. The ultimate goal and motivation for all is, of course, the nuclear many-body problem. It is therefore at least heartening that, after so many decades of study using the shell model and geometrical descriptions alone, a third approach has emerged which is fundamentally different in its outlook. Although attempts are made to understand and explain its predictions in the language of its predecessors, the origin of those predictions is algebraic and driven by the underlying symmetry inherent in the system. 


\section{Acknowledgment}

One of us (PVI) acknowledges financial support of the SERC through an advanced fellowship.

\section{References}

Alhassid Y, Engel J and Wu J 1984 Phys. Rev. Lett. 5317

Alhassid Y, Gürsey F and Iachello F 1983 Phys. Rev. Lett. 50873 1986 Ann. Phys. (NY) 167181

Alhassid Y and Iachello F 1989 Nucl. Phys. A 501585

Aprahamian A, Wu X, Fischer S M, Reviol W and Saladin J X 1993 IOP Conf. Ser. 132 585

Arima A, Harvey M and Shimizu K 1969 Phys. Lett. B 30517

Arima A and Iachello F 1975 Phys. Rev. Lett. 351069

Arima A and Iachello F 1976 Ann. Phys. (NY) 99253

Arima A and Iachello F 1978 Ann. Phys. (NY) 111201

Arima A and Iachello F 1979 Ann. Phys. (NY) 123468

Arima A, Otsuka T, Iachello F and Talmi I 1977 Phys. Lett. B 66205

Bahri C, Draayer J P and Moszkowski S A 1992 Phys. Rev. Lett. 682133

Baktash C, Nazarewicz W and Wyss R 1993a Nucl. Phys. A 555375

Baktash C, Winchell D F, Garrett J D and Smith A 1993b Nucl. Phys. A 557 145c

Balantekin B, Bennett J R, DeWeerd A J and Kuyucak S 1992 Phys. Rev. C 462019

Bauske I, Arias J M, von Brentano P, Frank A, Friedrichs H, Heil R D, Herzberg H-D, Hoyler F, Van Isacker P, Kneissl U, Margraf J, Pitz H H, Wesselborg C and Zilges A 1993 Phys. Rev. Lett. 71975

Bijker R and Kota V K B 1988 Ann. Phys. (NY) 187148

Bohle D, Kilgus G, Richter A, de Jager C W and de Vries H 1987 Phys. Lett. B 195326

Bohle D, Richter A, Steffen W, Dieperink A E L, Lo Iudice N, Palumbo F and Scholten O 1984 Phys. Lett. B 13727

Bohr A, Hamamoto I and Mottelson B R 1982 Phys. Scripta 26267

Bohr A and Mottelson B R 1969 Nuclear Structure Vol 1 (New York: Benjamin)

Bruce A M, Gelletly W, Colvin C G, Van Isacker P and Warner D D 1992 Nucl. Phys. A 5421

Byrski T, Beck F A, Curien D, Schuck C, Fallon P, Alderson A, Ali I, Bentley M A, 
Bruce A M, Forsyth P D, Howe D, Roberts J W, Sharpey-Shafer J F, Smith G and Twin P J 1990 Phys. Rev. Lett. 641650

Casten R F and Cizewski J A 1978 Nucl. Phys. A 309477

Casten R F, von Brentano P and Haque A M I 1985 Phys. Rev. C 311991

Casten R F and Warner D D 1988 Rev. Mod. Phys. 60389

Casten R F, Chou W T and Zamfir N V 1993 Nucl. Phys. A 555563

Cizewski J A, Casten R F, Smith G J, Stelts M L, Kane W R, Börner H G and

Davidson W F 1978 Phys. Rev. Lett. 40167

De Coster C and Heyde K 1991 Nucl. Phys. A 524 441; 529507 and refs. therein

De Coster C and Heyde K 1993 Phys. Lett. B 305322

Délèze M, Drissi S, Jolie J, Kern J and Vorlet J P 1993 Nucl Phys A 5541

Dieperink A E L, Scholten O and Warner D D 1987 Nucl. Phys. A 469173

Draayer J P 1993 Contemporary Concepts in Physics Volume 6 (edited by Casten R F, Harwood Academic) Chapter 7

Dudek J, Nazarewicz W, Szymanski Z and Leander G A 1987 Phys. Rev. Lett. 591405

Elliott J P 1985 Rep. Prog. Phys. 48171

Elliott J P and Dawber P G 1979 Symmetry in Physics Vols 1 \& 2 (Oxford: Oxford University Press)

Elliott J P and Evans J A 1981 Phys. Lett. B 101216

Elliott J P and Evans J A 1987 Phys. Lett. B 1951

Elliott J P, Evans J A and Long G L 1992 J. Phys. A 254633

Elliott J P, Evans J A and Van Isacker P 1988 Nucl. Phys. A 481245

Elliott J P and White A P 1980 Phys. Lett. B 97169

Evans J A, Elliott J P and Szpikowski S 1985 Nucl. Phys. A 435317

Evans J A, Van Isacker P and Elliott J P 1989 Nucl. Phys. A 489269

Faessler A, Nojarov R and Scholtz F G 1990 Nucl. Phys. A 515237 and refs. therein

Frank A, Alhassid Y and Iachello F 1986 Phys. Rev. A 34677

Frank A, Arias J M and Van Isacker P 1991 Nucl. Phys. A 531125

Frank A, Pittel S, Warner D D and Engel J 1986 Phys. Lett. B 182233

Frank A and Van Isacker P 1994 Algebraic Methods in Molecular and Nuclear Structure Physics (New York: Wiley-Interscience)

Frank A and Wolf K B 1984 Phys. Rev. Lett. 521737

Freeman S J, Chapman R, Durell J L, Hotchkis M A C, Khazaie F, Lisle J C, Mo J N, Bruce A M, Cunningham R A, Drumm P V, Warner D D, and Garrett J D 1989 
Phys. Lett. B 222347

1993a Nucl. Phys. A 554333

Freeman S J, Chapman R, Durell J L, Hotchkis M A C, Khazaie F, Lisle J C, Mo J N, Bruce A M, Cunningham R A, Drumm P V and Warner D D 1993b Nucl. Phys. A 55210

Gell-Mann M 1962 Phys. Rev. 1251067

Halse P, Elliott J P and Evans J A 1984 Nucl. Phys. A 417301

Hamamoto I and Åberg S 1984 Phys. Lett. B 145163

Hamamoto I and Åberg S 1986 Phys. Scripta 34697

Hamilton W D, Irbäck A and Elliott J P 1984 Phys. Rev. Lett. 532469

Harter H, von Brentano P and Gelberg A 1986 Phys. Rev. C 341472

Hartmann U, Bohle D, Guhr T, Hummel K-D, Kilgus G, Milkau U and Richter A 1987 Nucl. Phys. A 46525

Hecht K T and Adler A 1969 Nucl. Phys. A 137129

Iachello F and Arima A 1987 The Interacting Boson Model (Cambridge: Cambridge University Press)

Iachello F and Talmi I 1987 Rev. Mod. Phys. 59339

Iachello F and Van Isacker P 1991 The Interacting Boson-Fermion Model (Cambridge: Cambridge University Press)

Ikeda A and Shimano T 1993 Nucl. Phys. A 557 573c

Kneissl U 1992 Prog. Part. Nucl. Phys. 28331 and refs. therein

Leigh J R, Rowley N, Lemmon R C, Hinde D J, Newton J O, Wei J X, Mein J C, Morton C R, Kuyucak S and Kruppa A T 1993 Phys. Rev. C 47 R437

Lépine-Szily A, Obuti M M, Lichtenhäler Filho R, Oliveira J M Jr and Villari A C C 1990 Phys. Lett. B 24323

Lépine-Szily A, Oliveira J M Jr, Fachini P, Lichenthäler Filho R, Obuti M M, Sciani W, Steinmayer M K and Villari A C C 1992 Nucl. Phys. A 539487

Lipas P O, von Brentano P and Gelberg A 1990 Rep. Prog. Phys. 531355

Mauthofer A, Stelzer K, Gerl J, Elze Th W, Happ Th, Eckert G, Faestermann T,

Frank A and Van Isacker P 1986 Phys. Rev. C 341958

Nazarewicz W, Twin P J, Fallon P and Garrett J D 1990 Phys. Rev. Lett. 641654

Okubo S 1962 Prog. Theor. Phys. 27949

Otsuka T, Arima A and Iachello F 1978 Nucl. Phys. A309 1

Ratna Raju R D, Draayer J P and Hecht K T 1973 Nucl. Phys. A 202433 
Richter A 1988 Int. Conf. on Contemporary Topics in Nuclear Structure Physics (Cocoyoc, Mexico, June 9-14, edited by Casten R F et al.) 127

Richter A 1991 Nucl. Phys. A 522 139c and refs. therein

Scholten O, Heyde K, Van Isacker P, Jolie J, Moreau J, Waroquier M and J Sau 1985 Nucl. Phys. A 43841

Stephens F S, Deleplanque M A, Draper J E, Diamond R M, Macchiavelli A O, Beausang C W, Korten W, Kelly W H, Azaiez F, Becker J A, Henry E A, Yates S W, Brinkman M J, Kuhnert A and Cizewski J A 1990 Phys. Rev. Lett. 65 301

Sugawara-Tanabe K and Arima A 1988 Phys. Lett. B 206573

Sugawara-Tanabe K and Arima A 1989 Phys. Lett. B 229327

Thompson M J, Elliott J P and Evans J A 1987 Phys. Lett. B 195511

Van Isacker P, Elliott J P and Warner D D 1987 Phys. Rev. C 361229

Van Isacker P and Frank A 1989 Phys. Lett. B 2251

Van Isacker P, Heyde K, Jolie J and Sevrin A 1986 Ann. Phys. (NY) 171253

Van Isacker P, Lipas P O, Helimäki K, Koivistoinen I and Warner D D 1988

Nucl. Phys. A 476301

Warner D D 1991 Nucl. Phys. A 522 119c

Warner D D 1993 Contemporary Concepts in Physics Volume 6 (edited by Casten R F, Harwood Academic) Chapter 5

Warner D D and Bruce A M 1984 Phys. Rev. C 301066

Warner D D and Van Isacker P 1990 Phys. Lett. B 2471

Wesselborg C, von Brentano P, Zell K O, Heil R D, Pritz H H, Berg U E P, Kneissl U, Lindenstruth S, Seemann U and Stock R 1988 Phys. Lett. B 20722

Wilets L and Jean M 1956 Phys. Rev. C 102788

Wolf A, Warner D D and Benczer-Koller N 1985 Phys. Lett. B 1587

Wu C L, Feng D H, Chen X G, Chen J Q and Guidry M W 1986 Phys. Lett. B 168313

Wu J, Iachello F and Alhassid Y 1987 Ann. Phys. (NY) 17368

Wybourne B G 1974 Classical Groups for Physicists (New York: Wiley-Interscience)

Zamfir N V and Casten R F 1991 Phys. Lett. B 260265

Zawischa D and Speth J 1991 Z. Phys. A 33997

Ziegler W, Rangacharyulu C, Richter A and Spieler C 1990 Phys. Rev. Lett. 652515 
